\title{
Interpreting and predicting the yield of transit surveys: giant planets in the OGLE fields
}

\author{
F. Fressin ${ }^{1}$, T. Guillot ${ }^{2}$, V. Morello ${ }^{2}$, and F. Pont ${ }^{3}$ \\ 1 Observatoire de la Côte d'Azur, Laboratoire Gemini, CNRS UMR 6203, BP 4229, 06304 Nice Cedex 4, France \\ e-mail: fressin@obs-nice.fr \\ 2 Observatoire de la Côte d'Azur, Laboratoire Cassiopée, CNRS UMR 6202, BP 4229, 06304 Nice Cedex 4, France \\ 3 Geneva University Observatory, Switzerland
}

Received 19 January 2007 / Accepted 8 April 2007

\begin{abstract}
Transiting extrasolar planets are now discovered jointly by photometric surveys and by radial velocimetry, allowing measurements of their radius and mass. We want to determine whether the different data sets are compatible between themselves and with models of the evolution of extrasolar planets. We further want to determine whether to expect a population of dense Jupiter-mass planets to be detected by future more sensitive transit surveys. We directly simulate a population of stars corresponding to the OGLE transit survey and assign them planetary companions based on a list of 153 extrasolar planets discovered by radial velocimetry. We use a model of the evolution and structure of giant planets that assumes that they are made of hydrogen and helium and of a variable fraction of heavy elements (between 0 and $100 M_{\oplus}$ ). The output list of detectable planets of the simulations is compared to the real detections. We confirm that the radial velocimetry and photometric survey data sets are compatible within the statistical errors, assuming that planets with periods between 1 and 2 days are approximately 5 times less frequent than planets with periods between 2 and 5 days. We show that evolution models fitting present observational constraints predict a lack of small giant planets with large masses. As a side result of the study, we identify two distinct populations of planets: those with short periods $(P<10 \mathrm{~d})$, which are found in orbit only around metal-rich stars with $[\mathrm{Fe} / \mathrm{H}]>\sim-0.07$, and those on longer orbits $(P>10 \mathrm{~d})$, for which the metallicity bias is less marked. We further confirm the relative absence of low-mass giant planets at small orbital distances. Testing these results and the underlying planetary evolution models requires the detection of a statistically significant number of transiting planets, which should be provided over the next few years by continued ground-based photometric surveys, the space missions CoRoT and Kepler, and combined radial velocity measurements.
\end{abstract}

Key words. stars: planetary systems: formation - surveys - techniques: photometric - methods: data analysis stars: planetary systems

\section{Introduction}

Extrasolar planets are now routinely discovered orbiting solartype stars by radial velocimetry, but the discovery of transiting planets by photometric surveys is just beginning. Although still marginal, the late success of transit surveys has given an additional impulse to exoplanetology with the possibility of estimating the radius, density, and hence composition of extrasolar planets.

Quantitatively, to date we know 206 extrasolar planets with masses below $13 M_{\text {Jup }}$ (e.g. Udry et al. 2007; Butler et al. 2006). Among those, a list of 14 currently known transiting planets is presented in Table 1. These planets have been discovered by radial velocimetry followed by photometry for 3 of them, and by photometric surveys for the remaining 11 .

When considering the score of projects devoted to the detection of planets by transit photometry, the present harvest appears meager. The discrepancy between predictions (e.g. Horne 2001) and reality has been attributed to various factors such as: imperfect duty cycle, a reduced number of stars for which transiting planets are detectable (Gould et al. 2006), and the presence of correlated noises that can greatly limit the detectability of small planetary transits (Pont et al. 2006b). Several generic studies have been conducted to understand the yield of different transit surveys. Pepper \& Gaudi (2005) studied the optimization of transit searches as a function of the observational setup, the site properties, and the planet properties. Gillon et al. (2005) analyzed and compared deep field surveys, considering individual stellar ranges and observation windows, but did not include the effects of stellar crowding nor time-correlated noises.

Gould et al. (2006) studied the yield of OGLE survey (Udalski et al. 2002), the most successful so far in terms of number of transiting planets discovered, with a model populating the line of sight with stars drawn from the Hipparcos Catalogue. With that model, they estimated the proportion of stars with sensitivity to close-in giant planets to derive from OGLE results the frequency of planets as a function of their period. They find that the yield of the OGLE survey is globally consistent with the detections by radial velocimetry and with planet radii distributed between 1 and 1.25 jovian radii. The aim of the present work is to further test these data sets and the underlying physical model by a forward calculation of transit events with realistic stellar and planetary populations. In particular, we include upto-date models of the evolution and structure of Pegasids (closein extrasolar planets) based on models reproducing the observational constraints from known transiting planets (Guillot 2005; Guillot et al. 2006). As a consequence, we should be able to determine whether the presently known population of transiting planets represents the "tip of the iceberg", i.e. that many more small, dense, extrasolar giant planets exist and await discovery 
by the transit method, or whether it is relatively representative of the global population.

We first describe the model that is used to simulate transit surveys in general. In Sect. 3, we describe more particularly the OGLE surveys and the hypothesis chosen for their modelling. We then discuss the results of the simulation. A summary of the main conclusions and predictions for future transit surveys are provided in Sect. 5.

\section{Simulating transit surveys}

\subsection{General remarks}

The search for planets in transit in front of their star arose naturally with the discovery that a non-negligeable fraction of planets orbit very close to their stars. If orbital planes are randomly oriented, the probability that a planet will transit in front of its star at each orbital revolution is:

$\mathcal{P}_{\text {transit }} \simeq R_{\star} / a_{\text {planet }}$,

where $R_{\star}$ is the stellar radius, and $a_{\text {planet }}$ the planet's orbital semimajor axis. For systems such as $51 \mathrm{Peg} b$, this probability is close to $10 \%$. Because the probability for a solar-type star to possess such a Pegasid (i.e. a 51 Peg b-like planet, planets semi-major axis up to $0.1 \mathrm{AU}$ ) is about $0.5 \%$ (e.g. Marcy et al. 2005), 1 in 2000 solar-type stars should possess a transiting Pegasid. Using current results from radial velocity surveys and integrating over all periods, we estimate that about 1 in 1100 solar-type stars possess a transiting giant planet. Of course, depending on the magnitudes and field considered, giant stars may severely outnumber the dwarfs, so that in a real field, only one in, say, 3000 stars may harbor a transiting giant planet.

Because of the dependence on $a$, and period distribution, most of the transit events concerning giant planets occur for orbital periods between 1 and 5 days. The transits typically last for a couple of hours, as this quantity is weakly dependant on the orbital period $P$ :

$\tau_{\text {transit }}=1.82\left(\frac{P}{1 \text { day }}\right)^{1 / 3}\left(\frac{M_{\star}}{M_{\odot}}\right)^{-1 / 3}\left(\frac{R_{\star}^{\prime}}{R_{\odot}}\right) \mathrm{h}$,

where $R_{\star}^{\prime}$ is the length of the cord traced on the stellar disk by the planet's trajectory. (more precisely: $R_{\star}^{\prime}=R_{\star} \cos b+R_{\text {planet }}$, where $b$ is the impact parameter of the transit).

The depth of the transits themselves is given directly by the ratio of the planetary to the stellar disk surfaces:

$\mathcal{R}_{\text {transit }} \simeq\left(R_{\text {planet }} / R_{\star}\right)^{2}$.

This value is of order $1 \%$ for a Jupiter-size planet orbiting a Sun-like star. For an F-type star with radius $\sim 1.2 R_{\odot}$, the ratio decreases to $0.7 \%$. Furthermore, transiting giant planets discovered so far have radii between 0.72 and $1.44 R_{\text {Jup }}$ (see Table 1 ). Allowing for stellar radii to vary between 0.8 and $1.3 R_{\odot}$ (a typical range, in magnitude limited surveys), this implies that we should expect $\mathcal{R}_{\text {transit }}$ to vary between $0.3 \%$ and $3 \%$, for giant planets only. The lower limit is in reality even smaller because for detection purposes we have to account for the fact that planets also orbit stars that are in multiple systems (like HAT-P-1), and hence a dilution factor may apply. Although grazing transits are ignored in this simple analysis, they are included afterwards in our simulations.

Altogether this implies that in order to detect transiting giant planets, many thousands of dwarf stars have to be monitored over periods of weeks for a photometric precision reaching below a fraction of a percent on an equivalent integration time of about one hour. This is typically done by following a relatively dense stellar field over a long time with a stable telescope, and using a camera equiped with a good CCD camera.

\subsection{Principle of the simulations}

On paper, the simulation of the forward problem is simple: one has to generate a complete stellar field, or obtain it from observations, put it on a discrete grid (the CCD), include on probabilistic arguments the planetary companions, calculate lightcurves including the various sources of noise, and determine which events are detectable. This is the principle of CoRoTlux, a code we first developed to predict the transit yield of CoRoT space telescope (Baglin et al. 2002) and quantify the need for follow-up observations, which is here applied to the case of OGLE.

The interesting point of such a forward simulation is the possibility of including relatively easily fine details such as the fact that planets are found more frequently around metal-rich stars, or, on the basis of planetary evolution models, the fact that young planets orbiting close to bright stars will be larger than old planets orbiting smaller stars at larger orbital distances. This requires however that a relatively large number of physically relevant parameters (for example, the mass, size, metallicity, and age of the stars) be properly defined.

We further detail the assumptions that we made for these simulations by describing how we generate the stellar and planetary populations, and how we attempt to include realistic sources of noise.

\subsection{The stellar population}

\subsubsection{Main targets and background stars}

Stellar fields differ enormously in terms of densities and stellar populations. It is therefore most important to properly account for this in order to simulate any given transit survey.

It would be very appealing to use direct observations as much as possible to closely match the observed target fields. But as we will see hereafter, many different characteristics of the stars (stellar metallicity, age, and subtype ...) are required, and these are difficult to obtain with generic observations. We therefore adopt the following procedure:

- the observed stellar densities are obtained from stellar counts by magnitude, on the real stellar fields (see Sect. 3.1);

- the characteristics of the stars are obtained following a Monte-Carlo method using the output of the Besançon model of the galaxy (Robin et al. 2003) obtained for the proper location of the survey;

- where stellar counts are not available, or uncomplete (i.e. for faint stars), we use both stellar counts and characteristics from the Besançon model.

Specifically, we keep track of the following parameters obtained directtly from the Besançon model:

- the mass of each star, used to compute orbital parameters of the transiting object;

- the apparent magnitude of the star in the observed spectral range (the $I$ filter in the case of the OGLE survey);

- the $V$ magnitude of the star, important to qualify the confirmability of a transit event with radial velocimetry;

- the surface temperature of the star; 
Table 1. Known transiting planets by $2006^{\star}$.

\begin{tabular}{|c|c|c|c|c|c|c|c|c|c|}
\hline \# & Name & $\begin{array}{l}M_{\text {planet }} \\
{\left[M_{\text {Jup }}\right]}\end{array}$ & $\begin{array}{l}R_{\text {planet }} \\
{\left[R_{\text {Jup }}\right]}\end{array}$ & $\begin{array}{c}\text { Period } \\
\text { [day] }\end{array}$ & $\begin{array}{c}a \\
{[\mathrm{AU}]}\end{array}$ & $\begin{array}{c}M_{\star} \\
{\left[M_{\odot}\right]}\end{array}$ & $\begin{array}{l}R_{\star} \\
{\left[R_{\odot}\right]}\end{array}$ & $\begin{array}{l}T_{\text {eff }} \\
{[\mathrm{K}]}\end{array}$ & $\begin{array}{c}\text { Metallicity } \\
{[\mathrm{Fe} / \mathrm{H}]}\end{array}$ \\
\hline \multicolumn{10}{|c|}{ OGLE planets } \\
\hline 6 & OGLE-TR-10 & $0.63 \pm 0.14$ & $1.26_{-0.07}^{+0.07}$ & 3.10129 & 0.04162 & $1.18 \pm 0.04$ & $1.16 \pm 0.06$ & $6075 \pm 86$ & $0.28 \pm 0.10$ \\
\hline 2 & OGLE-TR-56 & $1.17 \pm 0.04$ & $1.32_{-0.06}^{+0.06}$ & 1.211909 & 0.0225 & $1.17 \pm 0.04$ & $1.32 \pm 0.06$ & $6119 \pm 62$ & $0.19 \pm 0.07$ \\
\hline 5 & OGLE-TR-111 & $0.52 \pm 0.13$ & $1.067_{-0.054}^{+0.054}$ & 4.0144479 & 0.047 & $0.81 \pm 0.02$ & $0.831 \pm 0.031$ & $5044 \pm 83$ & $0.19 \pm 0.07$ \\
\hline 3 & OGLE-TR-113 & $1.35 \pm 0.22$ & $1.09_{-0.03}^{-0.034}$ & 1.4324757 & 0.0229 & $0.78 \pm 0.02$ & $0.77 \pm 0.02$ & $4804 \pm 106$ & $0.15 \pm 0.10$ \\
\hline 4 & OGLE-TR-132 & $1.14 \pm 0.12$ & $1.18_{-0.07}^{+0.03}$ & 1.689868 & 0.0299 & $1.26 \pm 0.03$ & $1.34 \pm 0.08$ & $6210 \pm 59$ & $0.37 \pm 0.07$ \\
\hline \multicolumn{10}{|c|}{ Other transit survey planets } \\
\hline 7 & TrES-1 & $0.76 \pm 0.05$ & $1.081_{-0.029}^{+0.029}$ & 3.0300737 & 0.0393 & $0.89 \pm 0.035$ & $0.811 \pm 0.020$ & $5250 \pm 75$ & $-0.02 \pm 0.06$ \\
\hline 11 & TrES-2 & $1.28 \pm 0.07$ & $1.24_{-0.06}^{+0.09}$ & 2.47063 & 0.0367 & $1.08 \pm 0.08$ & $1.00 \pm 0.05$ & $5960 \pm 100$ & $0.15 \pm 0.03$ \\
\hline 10 & XO-1 & $0.90 \pm 0.07$ & $1.184_{-0.018}^{-0.028}$ & 3.941634 & 0.0488 & $1.0 \pm 0.03$ & $0.928 \pm 0.033$ & $5750 \pm 13$ & $0.015 \pm 0.03$ \\
\hline 12 & HAT-P-1 & $0.53 \pm 0.04$ & $1.36_{-0.09}^{+0.011}$ & 4.46529 & 0.0551 & $1.12 \pm 0.09$ & $1.15 \pm 0.09$ & $5975 \pm 45$ & $0.13 \pm 0.02$ \\
\hline 13 & WASP-1 & $0.867 \pm 0.073$ & $1.443_{-0.039}^{+0.039}$ & 2.519961 & 0.0382 & $1.15 \pm 0.09$ & $1.453 \pm 0.032$ & $6200 \pm 200$ & \\
\hline 14 & WASP-2 & $0.88 \pm 0.07$ & $1.038_{-0.05}^{+0.05}$ & 2.152226 & 0.0307 & $0.79 \pm 0.08$ & $0.813 \pm 0.032$ & $5200 \pm 200$ & \\
\hline \multicolumn{10}{|c|}{ Transiting planets discovered with Radial velocities } \\
\hline 9 & HD189733 & $1.15 \pm 0.04$ & $1.154_{-0.032}^{+0.032}$ & 2.218573 & 0.0313 & $0.82 \pm 0.03$ & $0.758 \pm 0.016$ & $5050 \pm 50$ & $-0.03 \pm 0.04$ \\
\hline 8 & HD149026 & $0.330 \pm 0.02$ & $0.726_{-0.064}^{+0.032}$ & 2.87598 & 0.042 & $1.3 \pm 0.1$ & $1.45 \pm 0.1$ & $6147 \pm 50$ & $0.36 \pm 0.05$ \\
\hline 1 & HD209458 & $0.657 \pm 0.006$ & $1.320_{-0.025}^{+0.024}$ & 3.52474859 & 0.047 & $1.09 \pm 0.09$ & $1.148 \pm 0.002$ & $6117 \pm 26$ & $0 \pm 0.02$ \\
\hline
\end{tabular}

$M_{\text {Jup }}=1.8986112 \times 10^{30} \mathrm{~g}$ is the mass of Jupiter. $R_{\text {Jup }}=71,492 \mathrm{~km}$ is Jupiter's equatorial radius.

OGLE-TR-10: Bouchy et al. (2005); Udalski et al. (2002); Konacki et al. (2005); Santos et al. (2006); Pont et al. (2006a).

OGLE-TR-56: Konacki et al. (2003); Udalski et al. (2002); Torres et al. (2003).

Bouchy et al. (2005); Santos et al. (2006); Pont et al. (2006a).

OGLE-TR-111: Pont et al. (2004); Santos et al. (2006); Udalski et al. (2002); Winn et al. (2007); Bouchy et al. (2005).

OGLE-TR-113: Bouchy et al. (2004); Udalski et al. (2002); Konacki et al. (2004); Gillon et al. (2006).

OGLE-TR-132: Bouchy et al. (2004); Udalski (2003); Moutou et al. (2004); Magain et al. (2007).

TRES-1: Alonso et al. (2004); Laughlin et al. (2005); Winn et al. (2007).

TRES-2: O'Donovan et al. (2006).

XO-1: McCullough et al. (2006); Holman et al. (2006); Wilson et al. (2006).

HAT-P-1: Bakos et al. (2006).

WASP-1: Collier Cameron et al. (2006); Shporer et al. (2007); Charbonneau et al. (2006).

WASP-2: Collier Cameron et al. (2006); Charbonneau et al. (2006).

HD-189733: Bouchy et al. (2005); Bakos et al. (2006).

HD-149026: Sato et al. (2005); Charbonneau et al. (2006).

HD 209458: Brown et al. (2001); Cody \& Sasselov (2002); Wittenmyer et al. (2005); Winn et al. (2005); Knutson et al. (2007).

\# is the label of planets in figures; they are ranked in the order of their discovery.

- the luminosity of the star, calculated from its absolute magnitude;

- the radius of the star, calculated from total luminosity and effective temperature.

The mass and effective temperature of the stars are distributed linearly around values given by the Besançon model (at $\pm 20 \%$ ). Figure 1 shows a simulated distribution of stars for the OGLE Carina field. The ensemble of dwarf stars with types F4 and later are highlighted as these represent targets for which planetary transit events are detectable, and, within observational limits, confirmable by radial velocimetry.

The metallicity distribution is obtained from the model of Nordström et al. (2004), which is based on the GenevaCopenhagen survey of the Solar neighbourhood. These authors find that the distribution of the metallicities $[\mathrm{Fe} / \mathrm{H}]$ is well approximated by a Gaussian function with a mean of -0.14 and a dispersion of 0.19 dex. We use this Gaussian distribution and choose to ignore possible dependencies between stellar parameters (e.g. masses, ages...) and the metallicities. (The link between stellar type and metallicity appears to be negligible for F4 and later types anyway (F. Thévenin, pers. communication 2007).)

\subsubsection{Binary and triple systems}

Multiple stellar systems are important especially because of the possibility that stellar eclispes mimic planetary transits (Brown 2003). However, we choose to defer this problem to a later article. Multiple systems are taken into account anyway because they can yield a dilution of the planetary transit events that makes them more difficult to detect. Planets may be present indifferently on the primary, secondary or tertiary components of a stellar system. (However, we find that only planets around the primary targets have a non-negligible chance of being discovered by current ground based photometric survey.)

Specifically, following Duquennoy \& Mayor (1991), we consider that $50 \%$ of the stars are binaries and $20 \%$ of those are ternaries. Multiple systems are considered as individual stars at the same position on the CCD. We choose to estimate their properties more simply than for the other stars, on the basis of DM91:

- We randomly add companions to the initial draw of primary stars, without changing their properties. The total mass and luminosity of each multiple system is thus slightly higher than initially. 

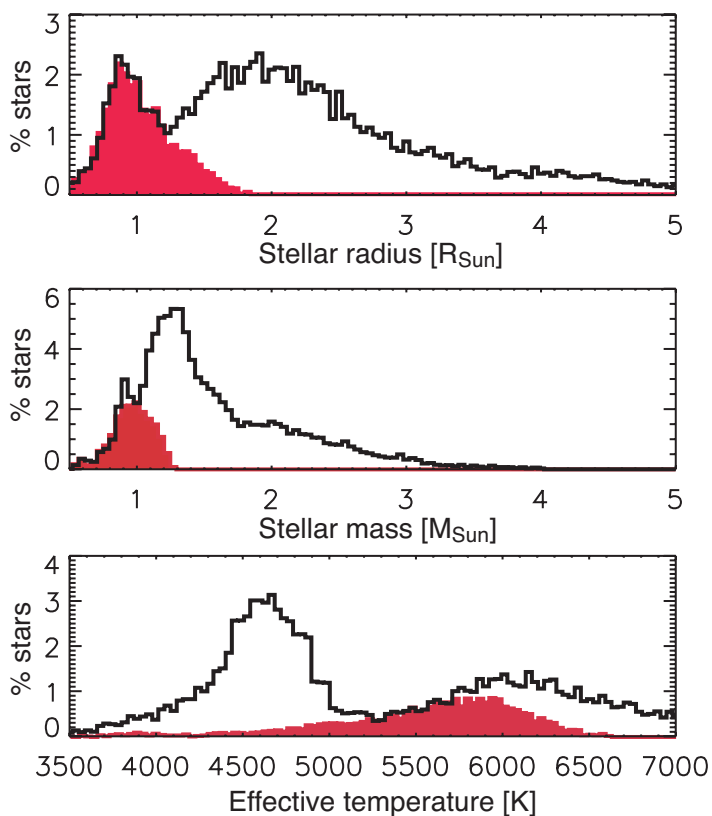

Fig. 1. From top to bottom: distribution functions for the radii, masses and effective temperatures for our fiducial stellar population corresponding to the simulated OGLE Carina field. The black line represents the ensemble of stars in the field. The filled red region is a subset for dwarf stars with stellar type F4 and later, as these are the only stars for which a transiting planet has a reasonnable chance of being detected by present-day transit surveys.

- The mass ratio (primary/secondary) is defined as a Gaussian of median value 0.23 and a full width at half maximum of 0.42 . Outside a range of 0.05 and 1 , we redraw the mass ratio.

- The radius is defined as $R_{\star}=R_{\odot}\left(M_{\star} / M_{\odot}\right)$ when $M_{\star} \leq M_{\odot}$ and $R_{\star}=R_{\odot}\left(M_{\star} / M_{\odot}\right)^{1 / 2}$ otherwise.

- The luminosity is assumed to be proportional to $M^{2}$ so that: $l_{\text {secondary }}=l_{\text {primary }}\left(M_{\text {secondary }} / M_{\text {primary }}\right)^{2}$.

- Other stellar parameters are calculated on the basis of these ones and of those of the primary component (same age, same distance, same metallicity).

- Triple components are treated with the same method as binaries, and are defined relatively to the primary star.

\subsection{The planetary companions}

With more than 200 planets known to orbit stars other than our Sun, we are beginning to have a rather precise view of at least part of this population. We can expect that biases on the detections are small in the case of massive planets (the mass of Saturn and more) and planets that are relatively close to their star (orbital distances smaller than $\sim 1 \mathrm{AU}$ ). These two conditions happen to match quite exactly the requirement for detectability by transit photometry, with one assumption: that only massive giant planets can have large radii. Although not proven, this assumption seems quite reasonnable.

Hence we choose to focus this study on this wellcharacterized population of objects. From the current list of 209 detected exoplanets, we select the ones discovered by radial velocities with mass higher than 0.3 Jupiter masses and known host star metallicity. Our list of planets includes 153 objects, to which we may add very-close in planets detected by transit photometry,
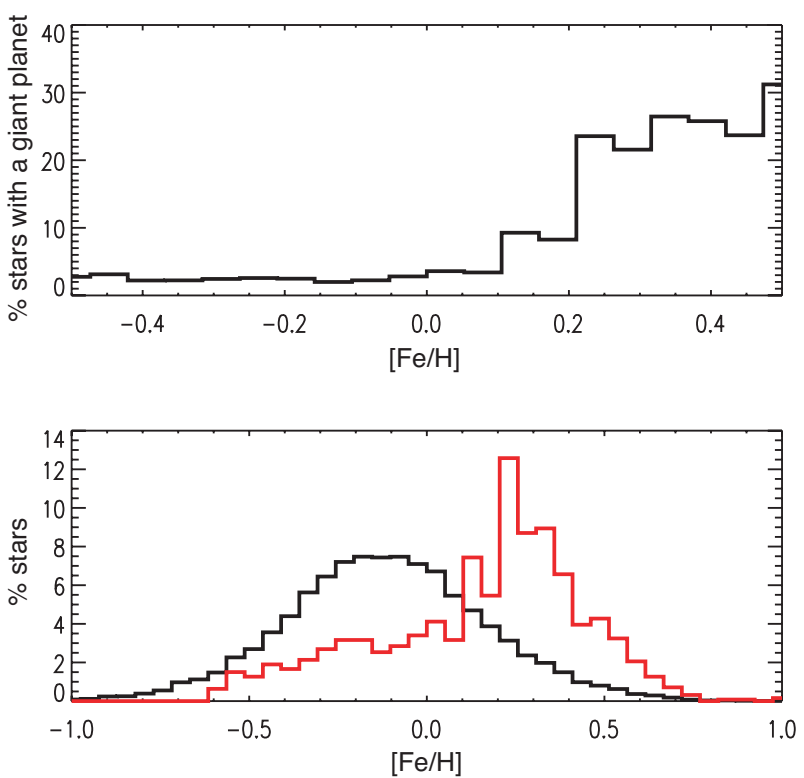

Fig. 2. Upper panel: probability for a solar-type star to possess a giant planet companion as a function of the stellar metallicity (from Santos et al. 2004). Lower panel: relative normalised distributions of stellar metallicities for stars in the field (black line), and for stars with a giant planet companion (red line).

as discussed below. We are using this list as representative of an unbiased sample of giant planets known from radial velocimetry, even though planetary distribution models may have been made from slighlty different samples.

\subsubsection{Planet incidence}

A first important step is the determination of the probability for a star to harbor a planet. As shown by numerous studies (Gonzalez 1998; Santos et al. 2004; Fischer \& Valenti 2005), this probability depends mostly on the metallicity of the parent star. Figure 2 shows one such probability function, as well as the result in terms of planet counts on a simulated stellar field.

In this work, we will use the dependency from Santos et al. (2004) shown in Fig. 2. Several points are to be considered however:

1. This probability relation is valid only for solar-like stars, i.e. F, G, K dwarf stars. Although there are strong indications that it may change for other stars (e.g. M dwarfs), we will assume it to hold independently of stellar properties. This assumption is sufficient because F, G, and K dwarf stars form by far the majority of stars with detectable planets in photometric surveys.

2. This relation has been calculated independently of the properties of the planetary companion, in particular orbital distance. Because in our case we are strongly biased towards short-period planets, the distribution may be different. This point will be considered in Sect. 4.4.

3 . The possibility of multiple planetary systems is not considered. This approach is justified because the probability that several planets belonging to the same system are transiting planets is small for giant planets. 


\subsubsection{Planetary masses and orbits}

The masses and orbital characteristics of the planet population are inferred almost entirely from the present radial-velocimetry surveys. This technique yields an accurate determination of the orbital period, and less accurately, of the eccentricity of the orbit. It also yields the value of the mass of the planetary companion times the sine of the orbital inclination from the knowledge of the mass of the parent star. With these values, we can then derive from a random inclination of the orbital planes the planets that are transiting and those that are not as well as the characteristics of their orbit. tities:

We test several approaches for the derivations of these quan-

- An analytical model: In this approach, we consider independantly the planet period and its mass. The period of the planet $\Pi$ follows the model of Brown (2003), the probability density $\mathcal{P}$ from a piecewise linear fit to the distribution $\mathcal{P}(\log \Pi)=\{0.509,0.165,0.533\}$ for three period intervals bounded by $\log \Pi=\{0.43,0.65,2.3,3.5\}$. The distribution in mass is linear in log from 0.3 to 10 Jupiter masses (Zucker \& Mazeh 2001). There is no dependency of these two parameters linked to metallicity.

- The radial velocity mass-period "carbon-copy" model: A second approach is to make direct use of the list of planets discovered by radial velocimetry. This is possible because in terms of masses and orbital periods the list is almost unbiased for the objects that we consider (massive enough to be above detection thresholds, and with periods much shorter than the lifetimes of the surveys). In this case, we select planets randomly in the RV list, and then allow for a small random deviation of their mass and period (a uniform deviation from $-20 \%$ to $+20 \%$ ) in order to avoid too much clustering on the same value. This is particularly important in the case of the period because of the importance of stroboscopic effects in planetary transits (e.g. Pont et al. 2005).

- The radial velocity mass-period-metallicity "carbon-copy" model: As a modification to the previous approach, we also consider using the metallicity entry in the RV list, because of correlations between metallicity and orbital period that are otherwise not taken into account (see discussion in section 4). We proceed slightly differently however than for the mass and orbital period because of the limitations caused by the finite number of planets in the RV list. In this case, we choose to split the list into two low- and high-metallicity lists, and then select the mass and periods in the relevant list. Our fiducial cutoff value is $[\mathrm{Fe} / \mathrm{H}]=-0.07$.

Figure 3 shows a comparison between observations, the carbon copy model and the analytical model. It is interesting to notice at this point that the carbon copy and analytical models are essentially indistinguishable in these diagrams. The differences with the observations arise only because of our choice to smear the masses and orbital periods when generating our planet population.

Last but not least, we have to consider the existence of planets that orbit extremely close to their star, with periods shorter than 2 days, as discovered by transit surveys (see Table 1). Companions with such short orbital periods have been discovered by radial velocimetry in two occasions: HD $41004 \mathrm{~b}$ and Gliese $876 \mathrm{~d}$, with respective masses 18.4 and 0.023 Jupiter masses. These objects are outside the mass range considered for this study, and therefore, there are no giant planets with periods shorter than 2 days in the present radial velocimetry list. In
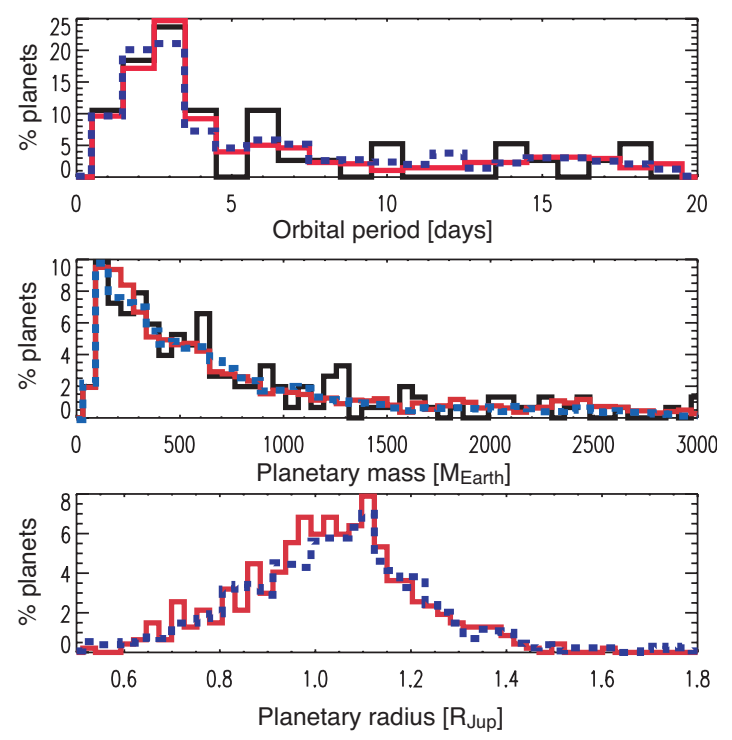

Fig. 3. From top to bottom, distributions of orbital periods, masses and radii, respectively, of the planets observed by radial velocimetry (black lines), simulated as part of the mass-period "carbon copy" model (red lines), and simulated as part of the analytical model (dotted blue lines) (see text).

order to account for these very close-in planets anyway, we add the planets with periods smaller than 2 days discovered by transit photometry to the list, but with a small tunable probability weight. The fiducial value of this parameter is set so that, on average, the planet list contains one and a half such planets (added to the list of $153 \mathrm{RV}$ planets described in Sect. 2.4). Tests on the effect of this parameter are presented in Sect. 4.3.4.

Our fiducial model is the mass-period-metallicity carbon copy model, which includes the addition of very-close in planets and it is that model which is used in all cases except where specified otherwise. Other approaches are also tested depending on the model to highlight particular points.

\subsubsection{Physical characteristics and the planetary evolution model}

Because we are focussing on planets more massive than Saturn, we expect most of them to be made of a significant amount of hydrogen and helium. These giant planets thus undergo a progressive contraction and cooling that depends on four quantities: their age, mass, the amount of flux the planet receives from the central star, and the global amount of heavy elements in the planet (e.g. Guillot 2005).

Models attempting to reproduce the radii of known transiting giant planets have however had problems in explaining the large radii of some of them (Bodenheimer et al. 2001; Guillot \& Showman 2002; Baraffe et al. 2005; Laughlin et al. 2005). Several possibilities have been proposed to explain the discrepancy. We can separate them into two categories:

- Mecanisms invoking chance configurations of the planetary orbits in the case of these anomalously large planets: this includes the tidal circularization of an eccentric orbit (Bodenheimer et al. 2001), and tidal dissipation for a planet locked in a Cassini spin-orbit resonnance with the star (Winn \& Holman 2005). 


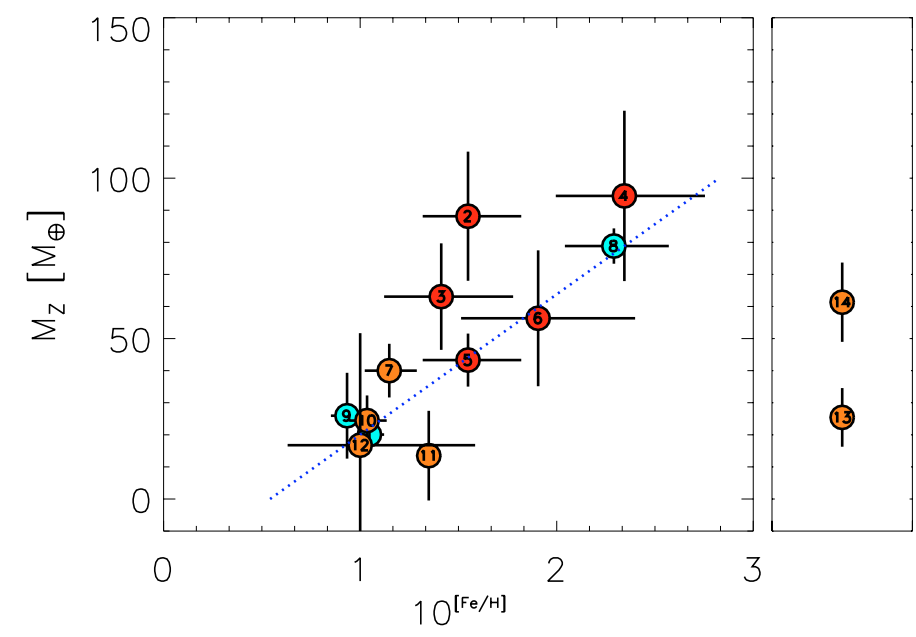

Fig. 4. Mass of heavy elements in transiting Pegasids known by 2006 as a function of the metal content of the parent star relative to the Sun. The mass of heavy elements required to fit the measured radii is calculated on the basis of evolution models including an additional heat source slowing the cooling of the planet. This heat source is assumed equal to $0.5 \%$ of the incoming stellar heat flux (Showman \& Guillot 2002). Horizontal error bars correspond to the $1 \sigma$ errors on the $[\mathrm{Fe} / \mathrm{H}]$ determination. Vertical error bars are a consequence of the uncertainties on the measured planetary radii and ages. The metallicity of recently discovered planets WASP-1 and WASP-2 (right panel) is not precisely known. The dotted line corresponds to a best fit model. (Adapted from Guillot et al. (2006).)

- Effects that would apply to all planets, including problems with the equations of state or opacities, and the dissipation by stellar tides of kinetic energy first generated in the atmosphere (Showman \& Guillot 2002).

The first mecanisms appear to have a low probability of occurence (Laughlin et al. 2005; Deming et al. 2005; Levrard et al. 2007). The second possibility therefore seems more likely, but requires in some cases the presence of relatively large masses of heavy elements to reproduce the observed radii.

A model-dependant estimate of the masses of heavy elements present in the currently known transiting Pegasids is shown in Fig. 4. This model relies on the hypothesis that $0.5 \%$ of the absorbed stellar flux is used to generate kinetic energy that is subsequently dissipated deep into the planetary interior (Guillot \& Showman 2002). As proposed by Guillot et al. (2006), there appears to be a correlation between the amount of heavy elements present in the planet and the metallicity of their parent star.

This correlation has to be ascertained, but we choose for our fiducial model to adopt a unique relation between metallicity and mass of heavy elements (treated as a central core in our calculations), corresponding to the dotted line in Fig. 4:

$M_{Z}=43.75 \times 10^{[\mathrm{Fe} / \mathrm{H}]}-23.7 M_{\oplus}$.

We limit the range of possible values of $M_{Z}$ to $\left[0,100 M_{\oplus}\right]$.

Similarly, we adopt a simple boundary condition for our evolution calculations:

$T_{1 \mathrm{bar}}=1.25 T_{\mathrm{eq} 0}$,

where $T_{1 \text { bar }}$ is the temperature at the 1 bar pressure level and $T_{\text {eq0 }}$ is the equilibrium temperature for a zero albedo (see Guillot 2005 for a description), calculated as a function of stellar effective temperature and radius and planet semi-major axis.

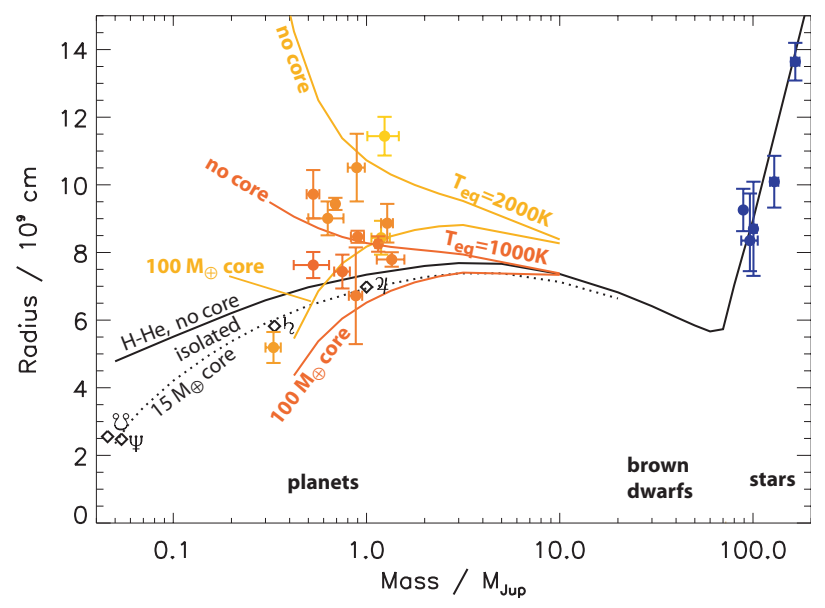

Fig. 5. Theoretical and observed mass-radius relations. The black line is applicable to the evolution of solar composition planets, brown dwarfs, and stars, when isolated or nearly isolated (as Jupiter, Saturn, Uranus, and Neptune, defined by diamonds and their respective symbols), after $5 \mathrm{Ga}$ of evolution. The dotted line shows the effect of a $15 M_{\oplus}$ core on the mass-radius relation. Orange and yellow curves represent the massradius relations for heavily irradiated planets with equilibrium temperatures of 1000 and $2000 \mathrm{~K}$, respectively, and assuming that $0.5 \%$ of the incoming stellar luminosity is dissipated at the center (see Sect. 2.4.3). For each irradiation level, two cases are considered: a solar-composition planet with no core (top curve), and one with a $100 M_{\oplus}$ central core (bottom curve). The transiting extrasolar giant planets for which a mass and a radius was measured are shown with points that are color-coded in function of the planet's equilibrium temperature. The masses and radii of very low mass stars are also indicated as blue points with error bars.

For simplicity, and because it yields only minor changes on the results, we further choose to neglect the time-dependence in the evolution calculations, and to adopt the equilibrium radius, or the $10 \mathrm{Gyr}$ solution, whichever occurs first.

Practically, planetary radii are obtained from interpolations in a table based on three parameters: the planetary mass ranging from 100 to $3000 M_{\oplus}$, the core mass from 0 to $100 M_{\oplus}$, and the equilibrium temperature from 100 to $2000 \mathrm{~K}$. Models were not calculated beyond these values of $T_{\mathrm{eq}}$ because of convergence problems. However we allowed for a slight extrapolation of the tables to $2600 \mathrm{~K}$ to account for rare extremely hot planets ${ }^{1}$.

Similarly, because of convergence problems for planets with small total masses and large core masses, we limited the mass of the core to $75 M_{\oplus}$ for planets with masses smaller than $180 M_{\oplus}$. More detailed work is required to better simulate this parameter space, including planets less massive than considered in this study.

Figure 5 shows examples of radii obtained for $T_{\text {eq }}=1000$ and $2000 \mathrm{~K}$, and core masses of 0 and $100 M_{\text {plus }}$, respectively, compared to available measurements.

\subsection{Modeling transit events and their detectability}

We now descibe how this population of stars, planets, and their interactions during transits are modelled to reproduce real observations.

\footnotetext{
1 An electronic version of the table is available at www.obs-nice.fr/guillot/pegasids/
} 


\subsubsection{PSFs and CCDs}

Each image of a star is spread by the atmosphere and by the telescope to grow to a specific size and shape when reaching the CCD in the focal plane of the telescope, the so-called point spread function (PSF). The CCD being composed of many discrete pixels, these PSFs are then effectively discretized, so that the signal to be analyzed for any given star is composed of many different lightcurves corresponding to the many pixels over the size of its PSF. A further complication arises from the fact that the stellar fields generally chosen by transit surveys are dense, so that many PSFs overlap. Recovering individual stellar light curves from real data is a complex problem. Two popular methods are aperture photometry (Stetson 1987) and image subtraction (Alard \& Lupton 1998). (An adaptation of the latter was used to extract the OGLE lightcurves.)

A refined simulation could include possible spatial and temporal variations of the PSFs, and a realistic data reduction pipeline. In our case, we choose to simplify the problem by relying on a posteriori analyses of real light curves to provide us with a global noise budget. We however include background stars because of the important effect of signal dilution.

In order to do so, we assume that the PSFs are Gaussian with a uniform, constant FWHM. (Non-Gaussian PSFs are not difficult to include but we tested in the OGLE case that for a fixed equivalent $F W H M$, they have a negligible effect on the resulting signal-to-noise ratio of simulated transit events). We consider for each target of the survey the global flux from the main star and the background stars in its neighborhood up to magnitude 22 in the spectral band of observation. The neighborhood zone for background stars is defined as a circle of diameter equal to 4 times the PSF's FWHM around the photocenter of each target star. Each background star whose photocenter is located in that zone is taken into account for the calculation of the global flux. The global flux is calculated as the sum of the pixels located at less than twice the $F W H M$ of the central star.

We thus simulate aperture photometry when image subtraction was used for OGLE (Udalski et al. 2002). The choice of the reduction algorithm indeed affects the sensitivity obtained from real observations. In our simulations, i.e. a relatively idealized case, it would have marginal effects since realistic noises are included a posteriori from the analysis of real lightcurves (see hereafter).

\subsubsection{Noise budget and event detectability}

We choose to separate noise sources into two categories:

- "White noise" sources, following Gaussian and Poisson laws. The main source of white noise is the photon noise of target stars and their background. The level of white noise for a given target star is obtained from the simulation of the flux of that star and its background in the photometric aperture.

- "Red noise", or systematic effects on photometry, that undergo temporal correlation. The structure of these systematics in the OGLE photometry have been explored in detail by Pont et al. (2006b). These noise sources are both instrumental (jitter and breathing of the CCD, frequency spectrum of stellar field moves on the camera, change of the PSF shapes accross the CCD during the night), and environmental (differential refraction and extinction, changes of airmass and sky brightness, temperature changes). Rather than trying to simulate instrumental and environmental noise sources accurately, which is difficult with the relatively poor knowledge we have of the time spectrum of their combined effects, we use the effective global 'red noise' measurements of OGLEIII survey real light curves mentionned in Pont et al. (2006b), which consider the combined effect of these noise sources.

Pont et al. (2006b) calculated that, in the presence of a mixture of white and red noise (i.e. accounting for photometric systematics), the detection threshold for a transit survey is well described by a limit on the signal-to-noise ratio defined as:

$S_{r}^{2}=\frac{d^{2} n^{2}}{\sum_{k=1}^{N \operatorname{tr}} n k^{2}\left(\sigma_{\mathrm{w}}{ }^{2} / n_{k}+\sigma_{\mathrm{r}}{ }^{2}\right)}$

where $N$ tr is the number of transits sampled and $n_{k}$ the number of data points in the $k$ th transit. $\sigma_{\mathrm{w}}$ and $\sigma_{\mathrm{r}}$ are the standard deviation of measurement points of white and red noises, respectively, $d$ is the event depth and $n$ the total number of measurement points during the transit. Specifically, we obtain $n_{k}$ by counting for each transit the number of observation points between the middle of ingress and the middle of egress.

Equation (6) makes the disctinction between "white" noise sources that decrease with $n^{1 / 2}$, where $n$ is the number of successive measurements, and "red" noise sources that are limited by temporal correlation. Pont et al. (2006b) indeed show that taking the red noise into account makes a large difference on the detection threshold - in general as well as in its dependence on the planet parameters - and that models based on the assumption of white noise can be poor approximations of the actual detection threshold.

\section{The OGLE survey: input parameters}

\subsection{Basic parameters and observational procedure}

The Optical Gravitational Lensing Experiment (OGLE) has done 6 observation campaigns looking for transiting planets towards different fields of view from 2001 (Udalski et al. 2002). It took place at the Las Campanas Observatory, Chile, using the $1.3 \mathrm{~m}$ Warsaw telescope and the 8k MOSAIC camera, with a total field of view of $0.34^{\circ 2}$. All observations were made through the I filter. We assume for our PSF simulation an average seeing of 1 arcsec.

We analyze in this work the first three OGLE-III observation campaigns dedicated to transit search, as their treatment, analysis, and follow-up (with current data processing pipelines) has been completed:

- OGLE-III-1 (June 12 to July 28, 2001, described in Udalski et al. 2002; Udalski 2002). More than 800 images of three fields in the direction of the galactic bulge were collected within 32 nights. The exposure time was $120 \mathrm{~s}$, and each field was observed every $12 \mathrm{~min}$.

- OGLE-III-2 (February 17 to May 22, described in Udalski (2003)). More than 1100 images of three fields located in the Carina region of the galactic disk were collected in 76 nights. The exposure time was $180 \mathrm{~s}$, and the temporal resolution was about $15 \mathrm{~min}$.

- OGLE-III-3 (February 12 to March 26, described in Udalski et al. (2004)). The photometric data were collected during 39 nights spanning the 43 days of the survey. Three fields of the galactic disk were observed with a time resolution of about $15 \mathrm{~min}$. The exposure time was $180 \mathrm{~s}$.

In this article, we will refer to these three observation campaigns respectively as "Bulge", "Carina", and "Centaurus" fields. 
Table 2. Fraction of stars suitable for transit detection.

\begin{tabular}{ccccc}
\hline \hline & \multicolumn{2}{c}{ Carina } & \multicolumn{2}{c}{ Bulge } \\
$V_{\max }$ & Gould 2006 & This work & Gould 2006 & This work \\
\hline 15.5 & 0.11 & 0.16 & 0.138 & 0.141 \\
16 & 0.14 & 0.16 & 0.125 & 0.128 \\
16.5 & 0.16 & 0.15 & 0.098 & 0.105 \\
17 & 0.16 & 0.15 & 0.068 & 0.080 \\
17.5 & 0.16 & 0.14 & 0.041 & 0.052 \\
\hline
\end{tabular}

The simulations include the real observation windows of each survey, as kindly provided by A. Udalski. For any transiting planet in the simulation, the number of effectively observed transits is used in Eq. (6).

In order to construct a realistic stellar population, we use the stellar counts per magnitude range obtained by Gould et al. (2006) based on OGLE-II data, which have calibrated photometry. We then randomly select that number of stars per magnitude from the Besançon model. In order to test the validity of our approach, we calculated the fraction of "stars for which transits are detectable" and compared it to the one determined by Gould. This fraction is defined for a given magnitude range as the number of stars around which a planet orbiting edge-on with $r=1.2 R_{\text {jup }}$ and $a=7.94 R_{\odot}$ can be detected, divided by the total number of stars of that magnitude. As shown by Table 2 , there is an excellent agreement between our results and those of Gould et al. (2006). Note however that for the global simulation, the complete star list is used as the above definition for suitable stars is restricted to planets of a given size and orbital distance.

We calculated the average flux for target stars, companions, and all the background stars near enough to contribute to the target PSF. We then checked that the average photon noise simulated for target stars at a given magnitude was close to real values obtained in OGLE light curves at given magnitude presented in Fig. 4 of Pont et al. (2006b).

\subsection{Modelling the detection threshold}

The candidates in the OGLE survey have been identified with the BLS transit-search algorith of Kovács et al. (2002). A subset of the candidates selected with cuts in the $\alpha$ and SDE parameters of the BLS were examined by eye, and only the best were included in the final list. Therefore, the selection threshold is mainly defined by subjective appreciation from an experienced specialist. Recently, Pont et al. (2006b) have pointed out that the effective detection threshold of ground-based transit surveys such as OGLE is importantly affected by correlated noise (photometric systematics). The subjective selection of candidates is in large part necessary because of the presence of this correlated noise, which produce many spurious detections near the threshold. Gould et al. (2006) chose to model the OGLE selection threshold with an $\alpha>12$ cut (alpha is equivalent to the signal-tonoise ratio of the transit signal assuming uncorrelated noise and homogeneous distribution of the data points in phase). Pont et al. (2006b) have included the effect of correlated noise in the signalto-noise calculation and found that the OGLE selection could be better described by a threshold of 8 on the signal-to-noise ratio of the transit signal calculated including correlated noise (" $S_{\mathrm{r}}$ " in their notation, see Sect. 2.5.2), and without the assumption of homogeneous coverage. While the two thresholds have similar effects on the global number of planet detection, they have a very different dependence on some parameters, such as planet period and host star magnitude. Since the objective or our study is to examine the detection statistics in a multi-dimensional parameter space, we use the Pont et al. (2006b) description of the OGLE detection threshold.

To calculate $S_{\mathrm{r}}$, one needs an assumption on the level of red noise present in the photometry. Following Pont et al. (2006b), we use a single-parameter description and assume $\sigma_{\mathrm{r}}=$ $3.6 \mathrm{mmag}$ in the Bulge fields, $\sigma_{\mathrm{r}}=3.1 \mathrm{mmag}$ in the Carina and Centaurus fields, and $\sigma_{\mathrm{r}}=2.1 \mathrm{mmag}$ in all fields after application of decorrelation algorithms.

\subsection{Confirmability of transit-like events with follow-up}

High-resolution spectra allow the confirmation of the planetary events if spectral lines are deep enough. Several scenarios make the follow up of candidates too difficult: early type stars have lines too weak and too broadened by rotation (type F4 and earlier). Stars with magnitudes $V>17.5$ are too faint for present instruments and telescopes. This is the limit at which observers estimated not being able to provide low-metallicity stars. Those stars having weaker lines, could also be difficult to follow correctly, but as planets are unlikely to be found near this kind of stars in our model, we did not take that parameter into account.

To simulate the feasability of follow-up, we only considered in CoRoTlux the stars matching the criteria $V<17.5$ and of type F4 and later.

\section{Results of the simulations}

We present hereafter runs for the three OGLE-III campaigns for the fields in the Galactic bulge, in Carina, and in Centaurus. In order to obtain a statistically significant population of detected planets, the simulations were run multiple times.

We first examine the consistency between the models and observations for relevant physical variables. In doing so, we choose to compare our model population to the global population of transiting planets discovered by OGLE and other surveys. There is a slight inconsistency in assuming that the parameter comparison is almost independant of the type of survey and observational strategy. In some cases, this is not true, and a clear distinction between the OGLE planets and the other detections has to be made.

We then discuss the problem of the detection statistics, whether observations and models are consistent, and whether a constraint on the (low) frequency of very close-in planets can be deduced.

\subsection{Deviation of OGLE planets from maximum likelihood of the simulations}

We use a Maximum-Likelihood (ML) technique in order to test whether model results and observations agree with each other. We do the tests in two-dimension spaces, in order to qualify possible correlation and exclusion zones. The ML technique is our method of choice as it is a powerful tool for fitting a model to a multi-dimentionnal independant-data distribution (Lyons 1986).

Instead of determining an approximate analytical law fitting our results, we use the results of a very large Monte-Carlo draw (1000 times the whole OGLE survey, corresponding to $\sim 9000$ planets) to get a map of the density of probability in each 2 -dimension grid. We bin our data on a $20 \times 20$ grid as a compromise between resolution of the models and characteristic 
variations of the parameters ${ }^{2}$. The probability of an event in each bin is considered equal to the normalized number of draws in that bin.

Figure 6 shows the logarithm of the probability that an event occurs in each of the $20 \times 20$ bins of the mass-radius diagram. The likelihood of a draw of several independant events is defined as the sum of the logarithms of the probabilities of these events. In order to compare our results to any $n$ real discoveries, we first estimate the standard deviation of any $n$-planets-randomdraw compared to the maximum likelihood of the model. We randomly select $n$ planets among the simulated detections and calculate the likelihood of this draw. We repeat this selection 1000 times in order to have the maximum likelihood and its standard deviation $\sigma$, then we compare the deviation of the likelihood of the $n$ real detecions calculated the same way in terms of $\sigma$. Henceforth, quantitative comparisons between the simulation results and the known planets are systematically given in the figure captions, whilst the text discusses qualitative comparisons and their implications. For the different figures showing the results of our simulation, we compare the distribution of planets over the detection threshold to the 5 OGLE planets. We also compare our results to the 11 planets discovered by all transit surveys, as their detection biases are similar to OGLE, and to the 14 planets, the radii of which are known (11 from transits and 3 from radial velocity surveys) to show how our model can reproduce the whole known population.

\subsection{Depth of the transit events and magnitude of the targets stars}

We first attempt to confirm whether the events detected by the model are consistent with those found in the OGLE fields. Figure 7 is a plot showing transit depth as a function of the magnitude of the primary star. Model results are considered detected when the signal-to-noise ratio is sufficient for a detection (see Sect. 3.2). We also show events that are considered photometrically detectable but very hard or impossible to confirm by radial velocimetry.

The figure evidently shows a good correlation between the black crosses and the red circles that indicate real detections by OGLE, with a range of transit depths and $\mathrm{V}$ magnitudes that is very similar between the models and the observations. Our models slightly overpredict the number of transit events around faint stars $(V \geq 17)$, but this may be due to the difficulty of the follow-up work for these targets. Overall, the agreement between models and observations is good.

\subsection{Compatibility of transit surveys with radial-velocimetry observations}

\subsubsection{Compatibility in the mass-period diagram}

Figure 8 compares the model and observated mass-period relation. As it is independant of the planetary evolution model, it is a direct test of the compatibility between the results of transit surveys and those of radial-velocimetry observations that drive our model results. Again, the comparison is very good, assuming a high-enough frequency of very-close in planets (see discussion in Sect. 4.3.4). One can note especially the absence of planets of relatively large mass (several times that of Jupiter) at short

\footnotetext{
2 Tests with different grids yield small variations of the results. As an example, the mass-radius deviation from maximum likelihood is respectively $0.67,0.65$, and $0.72 \sigma$ for $20 \times 20,30 \times 30$, and $40 \times 40$ grids.
}

orbital distances $(P<5$ days), and of detectable transit events for periods longer than $\sim 5$ days. This is due especially to the fact that only events with a relatively large number of observed transits are detectable, as required by the $S_{\mathrm{r}}$ threshold, which, given the day/night interruptions, imposes a constraint of a short orbital period. Note that this feature is not well reproduced by models in which the threshold is computed from white-noise only (Gould et al. 2006; Gillon et al. 2005).

\subsubsection{The OGLE yields with a fixed red noise level}

We have tested the efficiency of the fiducial model at quantitatively estimating the yield of transit surveys. Gillon et al. (2005) have also simulated OGLE yield in their generic study of multiple transit surveys, but with restrictive assumptions on transit detectability (only complete events matter for detection purposes) and without considering background stars and red noise, or using OGLE-fields specific stellar population. We also included in our simulations the recent RV follow-up that was done on Centaurus and Carina. We use unpublished information from the OGLE/ESO follow-up team, who found one promising planetary candidate among the Carina fields reprocessed with the systematics- removal algorithm from Tamuz et al. (2005) and none in the Centarus fields, with a magnitude limit near $V=17$ for the radial velocity follow-up. Table 3 compares the average number of planets detected for 1000 Monte-Carlo draws to real detections from the OGLE survey.

The total number of simulated discoveries obtained from this quantitative analysis is in good agreement with the real detections. The differences in the number of detections between the Carina and Centaurus surveys are mainly due to the lower duty cycle of the observations towards Centaurus. A red noise level fixed at $3.6 \mathrm{mmag}$ in the direction of the galactic bulge bans most hot Jupiter detections. The agreement between our quantitative result and the number of real detections is an indicator of the global efficiency of our approach (stellar and planetary distributions, evolution model and noise budget) for estimating transit survey yield.

\subsubsection{The OGLE yields with a variable red noise level}

So far, we have considered the level of red noise to depend only on the field considered. We attempt now to refine this by considering how the stellar density may affect it. Whereas most ground-based transit surveys have a global red noise level from 2 to 3.5 mmag (Superwasp: Smith et al. (2006), Monitor: Irwin et al. (2007), Hatnet: Pont \& ISSI team (2007) and OGLE), the causes of these noise levels seem different, with instruments ranging from $10-\mathrm{cm}$ wide field reflectors to deep-sky severalmeter telescopes. As seen from Table 3, the OGLE fields have different mean red noise levels $\left(\sigma_{\mathrm{r}}=3.6 \mathrm{mmag}\right.$ for the bulge and $\sigma_{\mathrm{r}}=3.1 \mathrm{mmag}$ for Centaurus and Carina before SYSREM), although the instrument and observational strategy were unchanged. Looking at what distinguishes these fields, it appears that the most significant difference is the stellar density and therefore the amount of crowding: the bulge field is about twice as dense as the Carina and Centaurus fields. Pont \& ISSI team (2007) raise the suspicion that the level of red noise depends strongly on the presence and characteristics of contaminating stars, because e.g. of their different colors and differential refraction in the atmosphere. It is hence natural to consider a red noise that depends on a crowding index. 

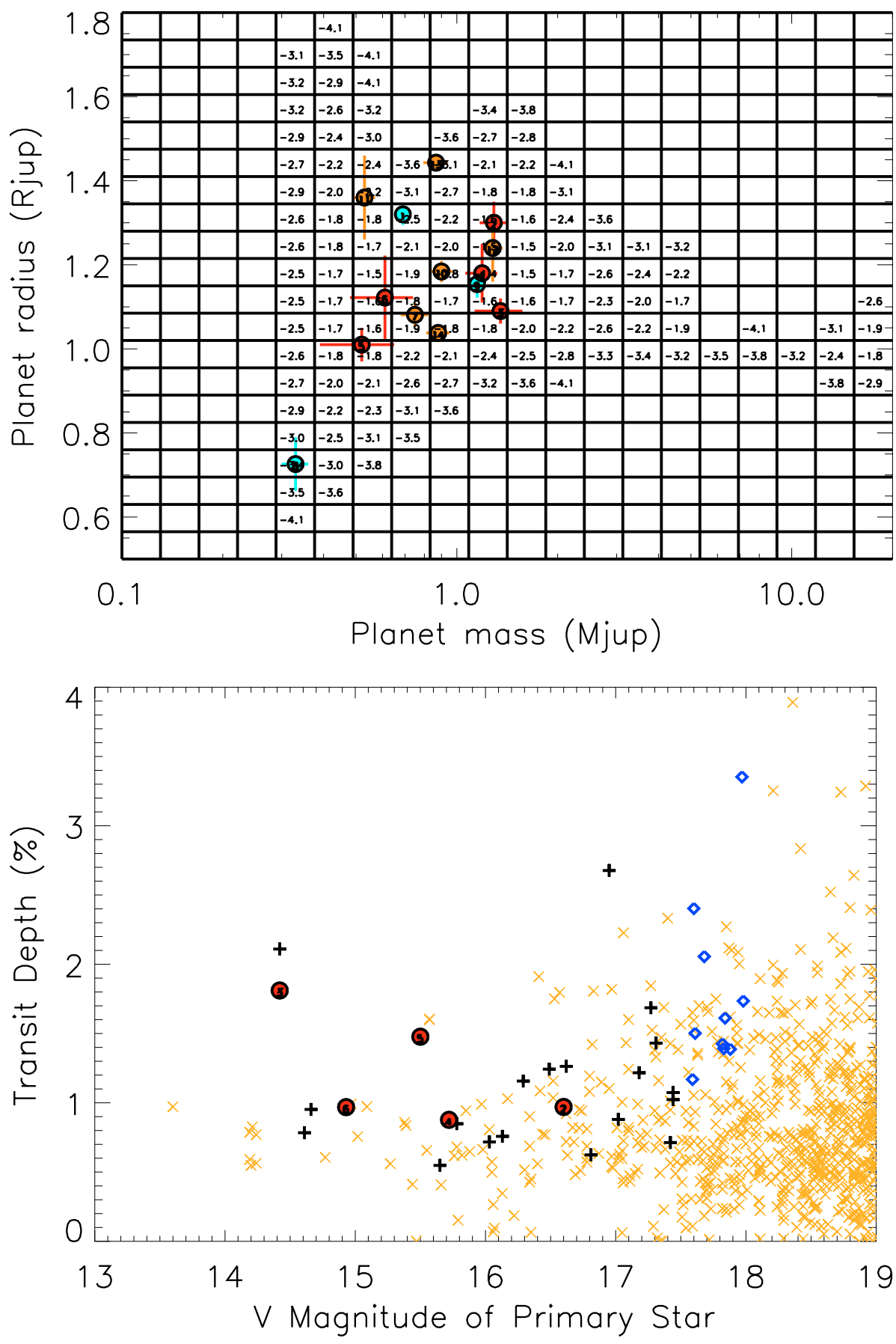

Fig. 6. Logarithm of the probability that a simulated detection event occurs in each one of the $20 \times 20$ bins of the mass/radius diagram. The likelihood of a multiple-events draw is the sum of the logarithms of the probabilities of the events of this draw. Bins without any occuring event in the large Monte-Carlo draw do not have any probability stated. The likelihood of a $n$-events draw is the sum of the probabilities of its $n$ events. In this mass-radius diagram, OGLE planets are shown as red circles, planets from other surveys are in orange, and planets from radial velocity surveys are in blue. The likelihood of the 5 OGLE discoveries as a result of a Monte-Carlo draw is -8.7 , the maximum likelihood is -7 and the standard deviation to maximum likelihood is 2.54 . Hence, the result of the OGLE planets mass-radius distribution is at $0.67 \sigma$ of the maximum likelihood of the model.

Fig. 7. Depth of the planetary transit events versus magnitude of the parent star in the $V$ band. The five confirmed OGLE detections are shown as circles. Model results are shown as black plusses for detectable events and orange crosses for events that are considered undetectable based on the photometric signal (see text). Blue diamonds correspond to events that would be detectable by photometry alone but that cannot be confirmed by radial velocimetry. Note that the model results correspond to 3 times the full OGLE campaign for more statistical significance. The OGLE planets depthmagnitude distribution is at $0.69 \sigma$ from the maximum likelihood of the model.
We define this crowding index as the fraction of the flux coming from background stars versus that from the target in the photometric aperture. Importantly, we do not consider stellar companions as contributing to the red noise because they are generally on the same CCD pixel as the target star and should affect the noise budget much less.

Figure 9 shows the differences of crowding index for the target stars with planetary transits (detectable or not) in simulations of the Carina and Bulge fields of view. The mean crowding index for target stars of $I<17$ is 0.11 in the Carina field and 0.233 in the Bulge field.

We can exclude the fact that all red noise is linked with contamination as many stars in the Carina fields are unblended by background stars but still show a high noise level.
In order to estimate of the influence of the crowding on the red noise level, we use the following simple relation between red noise level and crowding index:

$\sigma_{r}=\alpha \times F_{\mathrm{b}}+\beta$

where $F_{\mathrm{b}}$ is the fraction of total flux from background stars, determined on a star-by-star basis in our simulations, and $\alpha$ and $\beta$ are parameters to be determined. This is justified by the behaviour of the red noise seen for instance in SuperWASP, showing a linear increase as a function of background flux (Smith et al. 2006). In order to get the same mean red noise values as Pont et al. (2006b), we obtain $\alpha=0.4 \mathrm{mmag}$ and $\beta=2.65 \mathrm{mmag}$. This value of $\beta$ corresponds to the minimum red noise level obtained for non-contaminated stars in the OGLE fields. 


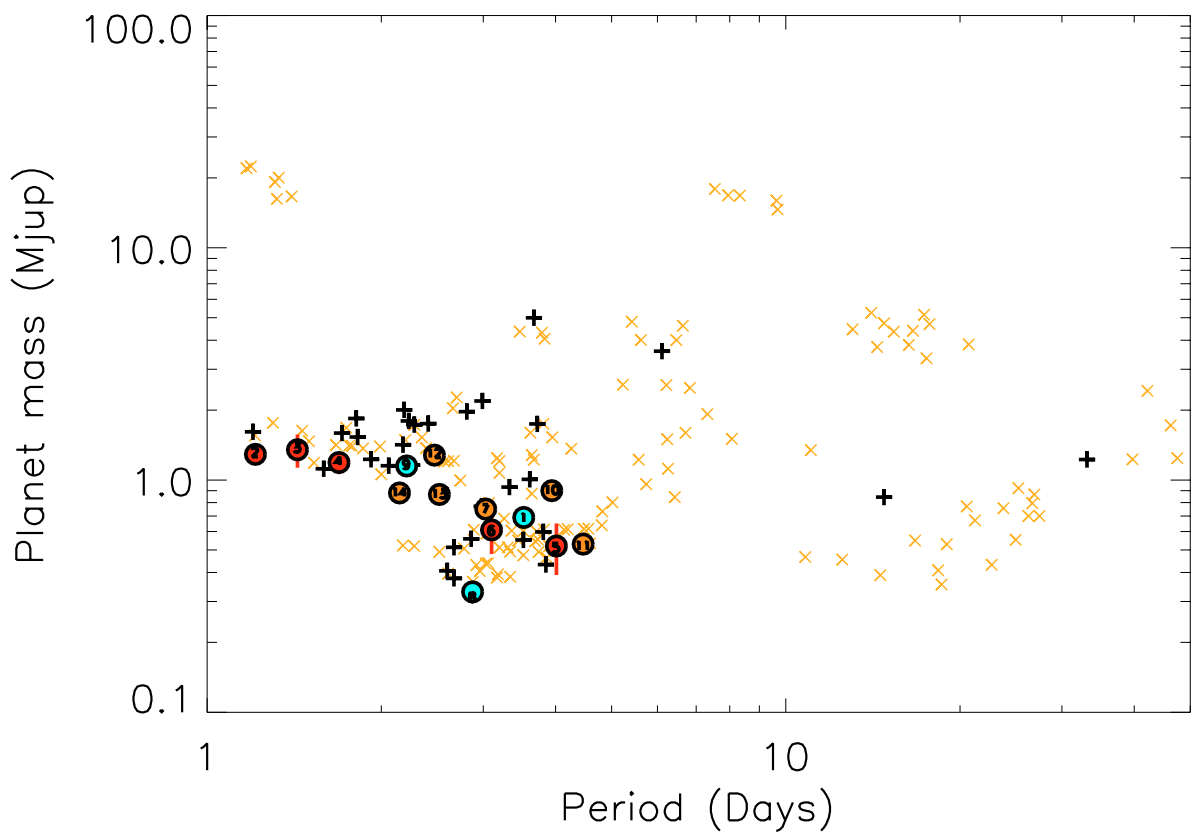

Fig. 8. Mass versus period of transiting giant planets. (OGLE planets are red circles, other transit surveys in orange, planets from radial velocity surveys in blue. Simulated planets detected: black plusses, under threshold: orange crosses). The OGLE planets mass-period distribution is at $0.62 \sigma$ from the maximum likelihood of the model $(0.72 \sigma$ considering the 11 planets discovered by transit surveys and $0.66 \sigma$ considering the 14 known planets).

Table 3. OGLE yields with fixed red noise level.

\begin{tabular}{ccccccc}
\hline \hline $\begin{array}{c}\text { Field } \\
\text { of view }\end{array}$ & $\begin{array}{c}\text { Mean red } \\
\text { noise level }\end{array}$ & $\begin{array}{c}\text { RV follow-up } \\
\text { to Vmag }\end{array}$ & \multicolumn{4}{c}{ Number of planets } \\
& & & & \multicolumn{4}{c}{$\begin{array}{c}\text { simulated with } \\
\text { VHJ added }(P<2 \text { days })\end{array}$} \\
\hline Bulge & 3.6 & 17.5 & 2 & 0.4 & 0.6 & 0.9 \\
\hline Carina $\begin{array}{c}\text { original } \\
\text { updated }\end{array}$ & 3.1 & 17.5 & 3 & 3.4 & 4.1 & 4.8 \\
\hline Centaurus & 2.1 & 17.5 & $+(0-1)$ & +1.1 & +1.1 & +1.1 \\
\hline Total & 3.1 & 17.0 & 0 & 1.4 & 1.8 & 2.2 \\
\hline
\end{tabular}

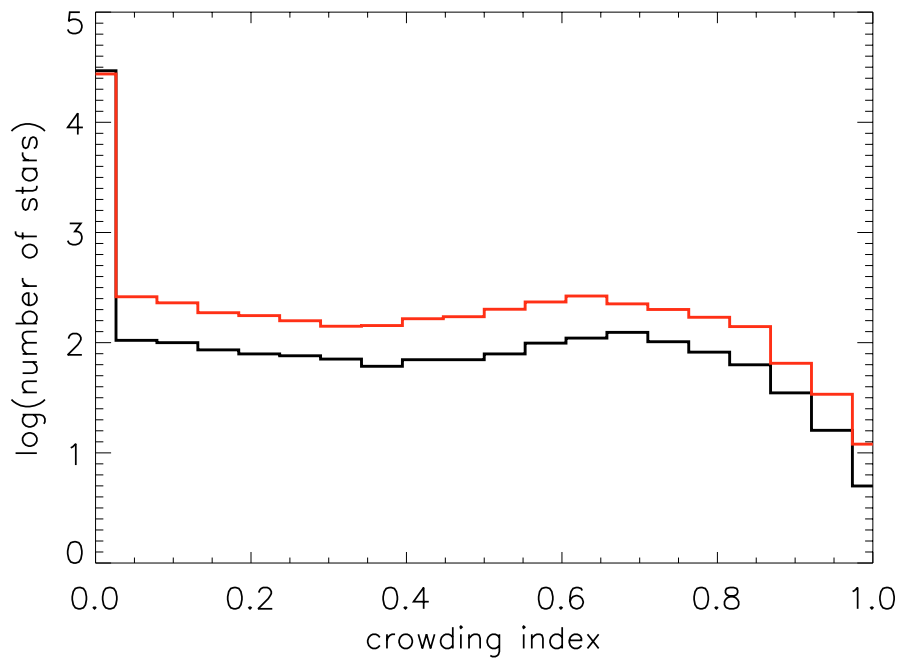

Fig. 9. Distribution of the crowding index (see text) of target stars in Carina (black) and in the bulge (red).

Table 4 shows the new number of detections when considering this crowding-dependant red noise level. Compared to Table 3, the number of detections is found to be essentially unchanged for the Carina and Centaurus fields, but it increases by a factor $\sim 3$ for the bulge field. This result is more satisfactory because in the previous case, only $\sim 5 \%$ of the simulations would yield the detection of 2 planets in the bulge, as observed.

\subsubsection{Models, observations and the frequency of very close-in planets}

As discussed in Sect. 2.4.1, three OGLE planets have orbital periods shorter than 2 days and thus belong to a class of objects yet to be detected by radial velocimetry. So far, we have added one such planet (on average) to our carbon copy list of nearly 200 radial velocimetry planets. In Sect. 4.3 , we have shown that with this assumption, radial-velocity and photometric transit surveys are compatible. We now test the range of frequencies of very close-in planets for which this remains true.

In order to do so, we compute the deviation from maximum likelihood in the mass-radius diagram like in Sect. 4.3, as a function of the number of planets having a period of less than 2 days added to the RV list. The result is presented in Fig. 10 and shows that a good match is obtained by adding 1 to 3 short-period planets. Larger numbers are also possible from the point of view of the transit surveys, but would conflict with their non-detection by radial-velocimetry. Adding the other transiting planets discovered thus far yields smaller probabilities of occurence of these short-period planets, but not by significant amounts.

All in all, and assuming that the radial velocity planets sample is unbiased, we constrain the fraction of main-sequence late stars orbited by very hot giant planets with orbital periods of less 
Table 4. OGLE yields with variable red noise level

\begin{tabular}{|c|c|c|c|c|c|}
\hline \multirow{4}{*}{$\begin{array}{l}\text { Field } \\
\text { of view }\end{array}$} & \multirow{4}{*}{$\begin{array}{l}\text { RV follow-up } \\
\text { to } V \text { mag }\end{array}$} & \multicolumn{4}{|c|}{ Number of planets } \\
\hline & & \multirow[t]{3}{*}{ detected } & \multicolumn{3}{|c|}{ simulated with } \\
\hline & & & 0 & 1.5 & 3 \\
\hline & & & \multicolumn{3}{|c|}{ VHJ added ( $P<2$ days) } \\
\hline Bulge & 17.5 & 2 & 1.2 & 1.6 & 2 \\
\hline original & 17.5 & 3 & 3.6 & 4.3 & 4.9 \\
\hline updated & 17.5 & $+(0-1)$ & +1.1 & +1.1 & +1.1 \\
\hline Centaurus & 17 & $\overline{0}$ & 1.3 & 1.9 & 2.3 \\
\hline Total & & $5-6$ & 7.2 & 8.9 & 10.3 \\
\hline
\end{tabular}

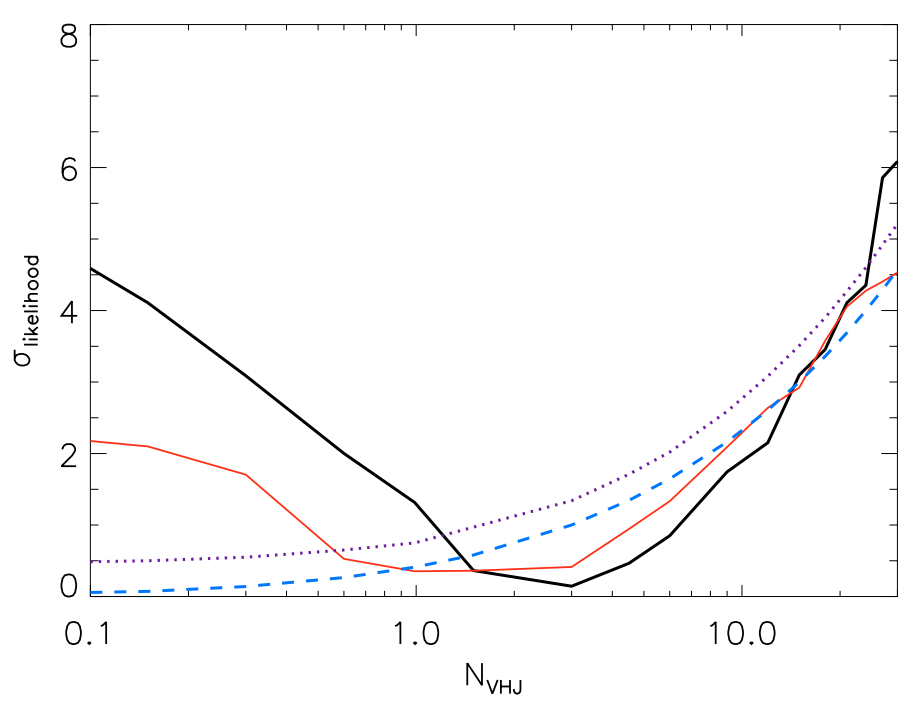

Fig. 10. Deviations from a maximum likelihood obtained as a function of $N_{\mathrm{VHJ}}$, the number of very hot jupiter of orbital periods shorter than 2 days added to the radial velocities carbon-copy list. Thick line: Deviation from the maximum likelihood obtained in the mass-radius diagram for the OGLE planets. Thin line: same deviation but when compared to the ensemble of planets. Dashed line: standard deviation obtained from a comparison between the number of simulated planets and the number of detected ones for the OGLE survey (see Table 4). Dotted line: standard deviation obtained from the non-detection of these very close-in planets by radial-velocimetry.

than 2 days to be $(1 / 1265)\left(1_{-0.33}^{+0.33}\right)$ at a $60 \%$ confidence level or $(1 / 1265)\left(1_{-0.5}^{+0.83}\right)$ at a $90 \%$ confidence level.

The distribution of planets in period between 2 and 5 days is directly obtained from the metallicity-linked distribution (Santos et al. 2004) and the RV planets sample. Adding the distribution we found for planets between 1 and 2 days, we obtain a fraction of $(1 / 215)$ late main-sequence stars orbited by planets in the 1-to-5-day period range, in good agreement with the results obtained in Gould et al. (2006), who obtained $(1 / 220)\left(1_{-0.45}^{+1.10}\right)$. Similarly, the distribution we obtain by cutting this sample into two parts with the cut-off at 3 days is compatible, showing:

- a slightly higher fraction of really short-period planets (13 days) of $(1 / 560)$ instead of $(1 / 710)\left(1_{-0.54}^{+1.10}\right)$ at a $90 \%$ confidence level in Gould et al. (2006);

- a similar fraction of short-period planets (3-5 days) of $(1 / 350)$ instead of $(1 / 320)\left(1_{-0.59}^{+1.39}\right)$ at a $90 \%$ confidence level in Gould et al. (2006).

The results presented hereafter use the variable red noise level approach, and an RV planet list that is complemented with, on average, 1.5 very-close in planets with periods $P<2$ days taken from the OGLE detections.

\subsection{The metallicity of the stars harboring transiting planets}

We now compare the metallicity of the parent stars for our observed and modelled populations. A first test using the analytical scenario for the radial-velocity population (Fig. 11) yields a clearly different metallicity distribution, with most of the transiting planets observed around low-metallicity stars. We verified that this problem occurs independantly of the assumed stellar metallicity distribution, for any realistic stellar population. It arises fundamentally because the global metallicity bias as obtained by Santos et al. (2004) or Fischer \& Valenti (2005) is not strong enough to compensate for the rarity of very metal-rich stars in the Galaxy.

As seen in Fig. 12, the problem disappears when one considers the carbon-copy model. Thus, we are led to an important conclusion, that the metallicity distribution of pegasids (periods shorter than 10 days) is fundamentally different from the global exoplanet population. More specifically, there are no Pegasids orbiting F, G, K stars with metallicities smaller than $[\mathrm{Fe} / \mathrm{H}]=$ -0.07 . This has strong consequences for planet formation models (see also Guillot et al. 2006). This work shows that this conclusion is robust, and is needed to explain the results of the photometric surveys.

A finer examination of Fig. 12 shows that while our model planets globally reproduce the metallicity of the ensemble of transiting planets, OGLE stars with planets are on average $\sim 0.1$ dex more metal-rich.

This can tentatively be explained with a metallicity gradient in the galaxy for OGLE TR-10 $([\mathrm{Fe} / \mathrm{H}]=0.28 \pm 0.10)$ and OGLE TR-56 $([\mathrm{Fe} / \mathrm{H}]=0.19 \pm 0.07)$, the two planets discovered in the direction of the galactic bulge. The study of galactic cepheids by Andrievsky et al. (2004) shows a metallicity gradient as a function of distance to the galactic center. In the $[6.6,10.6] \mathrm{kpc}-$ range distance from galactic center, this study finds a linear relation between $[\mathrm{Fe} / \mathrm{H}]$ and galactocentric distance $R_{\mathrm{G}}$ :

$[\mathrm{Fe} / \mathrm{H}]=-0.044( \pm 0.004) R_{\mathrm{G}}+0.363( \pm 0.032)$.

Following that relation, the two stars with planets discovered in the direction of the galactic bulge both at a distance around $1500 \mathrm{pc}$ would thus be in a 0.04 dex more metal rich region than the solar neighborhood.

Concerning the high metallicity of stars with transiting planets discovered by OGLE in the Carina region, we do not have any reason to think that the metallicity distribution would be different from the solar neighborhood. Our only hypothesis is a low-probability draw for metallicity for the 3 OGLE-Carina planets. 

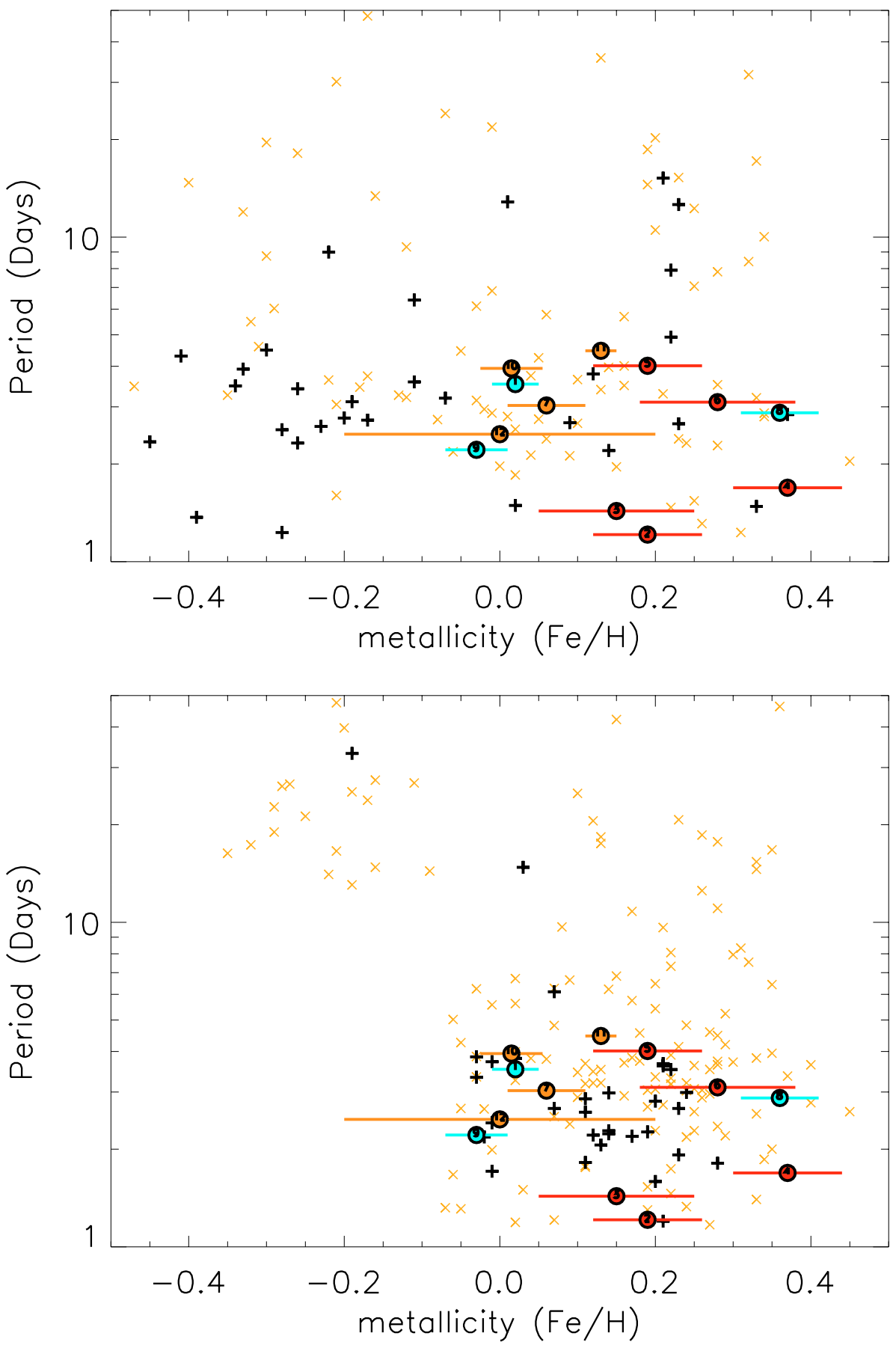

Fig. 11. Period of transiting exoplanets versus metallicity of their parent star. The model is based on analytic relations for the mass and period distributions of planetary companions (see Sect. 2.4.2). (OGLE planets are red circles, other transit surveys in orange, planets from radial velocity surveys in blue. Simulated planets detected: black plusses, under threshold: orange crosses). The OGLE planets periodmetallicity distribution is at $2.94 \sigma$ from the maximum likelihood of the model $(2.51 \sigma$ considering the 11 planets discovered by transit surveys and $2.63 \sigma$ considering the 14 known planets).

Fig. 12. Period of transiting exoplanets versus metallicity of their parent star. The figure differs from Fig. 11 in that our fiducial model, i.e. the mass-period-metallicity "carbon-copy" model is used (see Sect. 2.4.2). (OGLE planets are red circles, other transit surveys in orange, planets from radial velocitiy surveys in blue. Simulated planets detected: black plusses, under threshold: orange crosses). The OGLE planets period-metallicity distribution is at $0.76 \sigma$ from the maximum likelihood of the model $(0.36 \sigma$ considering the 11 planets discovered by transit surveys and $0.39 \sigma$ considering the 14 known planets) .

\subsection{Atmospheric potential energy and orbital distances}

Because evaporation may affect the planet population, it is instructive to check whether the potential energy of the atmosphere and the orbital period, two crucial quantities for this process (e.g. Lecavelier des Etangs et al. 2004), also possess a relatively consistent distribution. We first test the behavior of the analytical model for the distribution of planets (Fig. 13). This results in a prediction of many planets with large radii (small values of the potential energy for atmospheric escape) at small orbital distances, in patent contradiction with the observations.

The problem mostly disappears with the carbon-copy model: Fig. 14) shows that in this case, although we do not obtain a linear correlation between the two variables, we get detections in the right area of the diagram. This is explained as stemming from:

- The absence of low-mass planets at small orbital distances, with a possible limiting relation between these two quantities (Mazeh et al. 2005);

- The difficulty in detecting planets with larger values of potential energy per unit mass (smaller radii) at large orbital distances - although we predict that some of these should be detected by future transit surveys.

Our results strengthen the case for the existence of a relation between mass and orbital distance for short-period planets, as advocated by Mazeh et al. (2005): Indeed, the analytic model which is characterized by the presence of small mass planets at small distances yields a distribution of detectable planets 

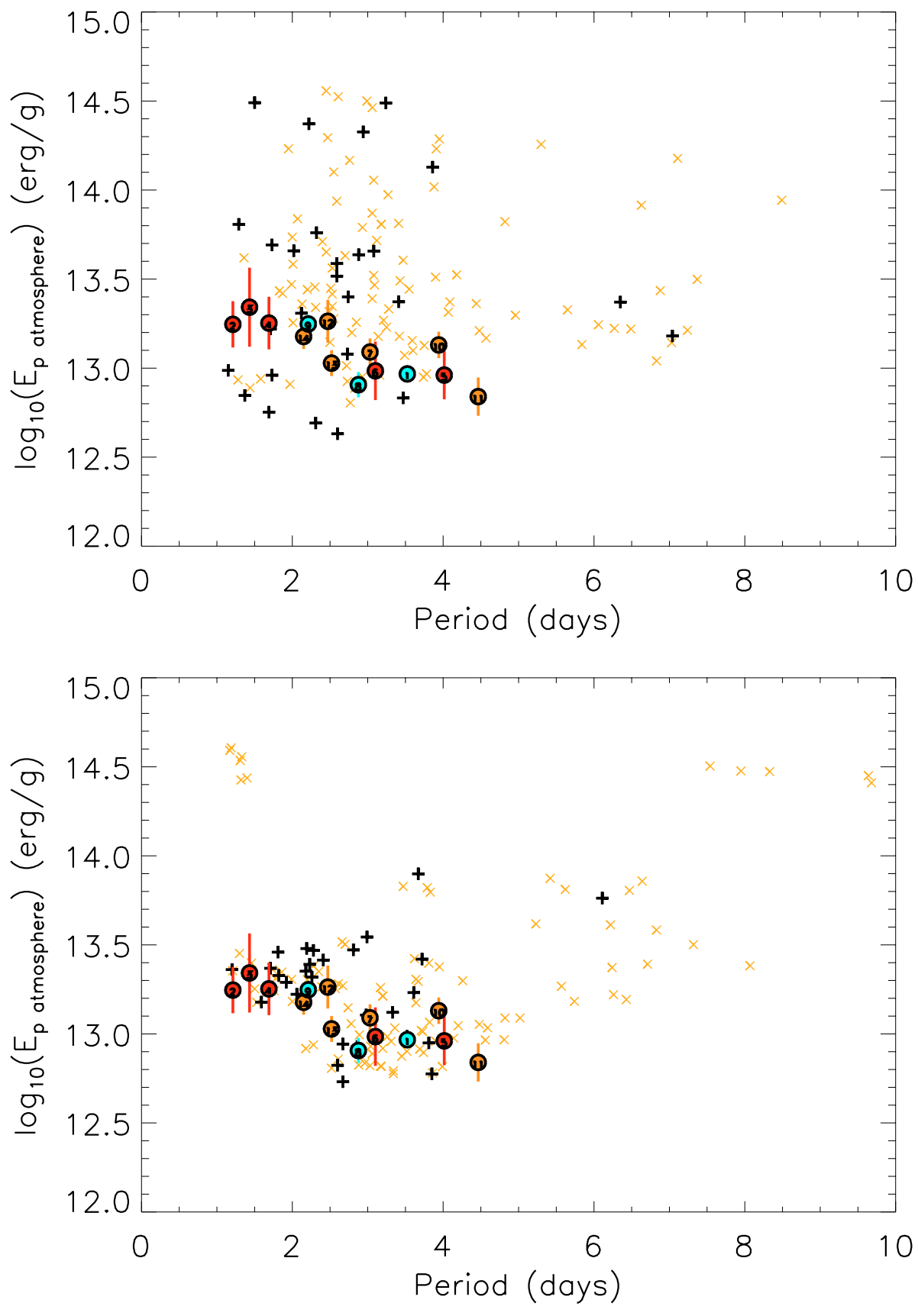

Fig. 13. Potential energy per unit mass $\left(E_{p}=\right.$ $G M / R$ ) versus orbital period of transiting planets. (OGLE planets are red circles, other transit surveys in orange, planets from radial velocity surveys in blue. Simulated planets detected: black plusses, under threshold: orange crosses). Observations are compared to models based on the analytical relations for the mass and period distribution of planetary companions (see Sect. 2.4.2). The OGLE planets' energy-period distribution is at $2.18 \sigma$ from the maximum likelihood of the model ( $1.86 \sigma$ considering the 11 planets discovered by transit surveys and $2.47 \sigma$ considering the 14 known planets).

Fig. 14. Potential energy per unit mass versus orbital period of transiting planets. The figure is similar to Fig. 13, except for the fact that our fiducial model is used (see Sect. 2.4.2). (OGLE planets are red circles, other transit surveys in orange, planets from radial velocity surveys in blue. Simulated planets detected: black plusses, under threshold: orange crosses). The OGLE planets energy-period distribution is at $0.55 \sigma$ from the maximum likelihood of the model. $(0.84 \sigma$ considering the 11 planets discovered by transit surveys and $0.66 \sigma$ considering the 14 known planets).

that is significantly different from the observations (Fig. 13). Our carbon-copy model that includes implicitely this correlation does not (Fig. 14).

\subsection{Planetary radii and stellar irradiation}

Radius and stellar irradiation (or equivalently equilibrium temperature) should be positively correlated, as a planet with a higher irradiation dose will tend to cool and contract more slowly than one that endures less stellar insolation. As Fig. 15 shows, the correlation exist, but is weak, and with a signficant scatter. This is well reproduced by the model.

However, it can be noted that HD 149026 b lies away from the cloud of points. In general, we find that our fiducial model generates few points in this region. This can be easily accounted for by slightly modifying the metallicity-core mass relation to allow for larger masses. As planets of small masses and large core masses are more difficult to model anyway, we chose not to attempt fine-tuning the model to this level of detail. This should be postponed for further studies, especially with the discovery of more Saturn-mass transiting planets.

\subsection{The mass-radius relation}

We have checked that our fiducial model predicts the detection of transiting planets with properties that are globally consistent with the observations. We can now examine in more detail the mass-radius relation thus obtained, as it is directly tied to assumptions on the compositions and evolutionary models of exoplanets. The predictions also have implications for transit surveys as it is not clear whether they have detected only the "tip of the iceberg", ie the few largest giant planets while many smaller ones would lie undetected or not. 


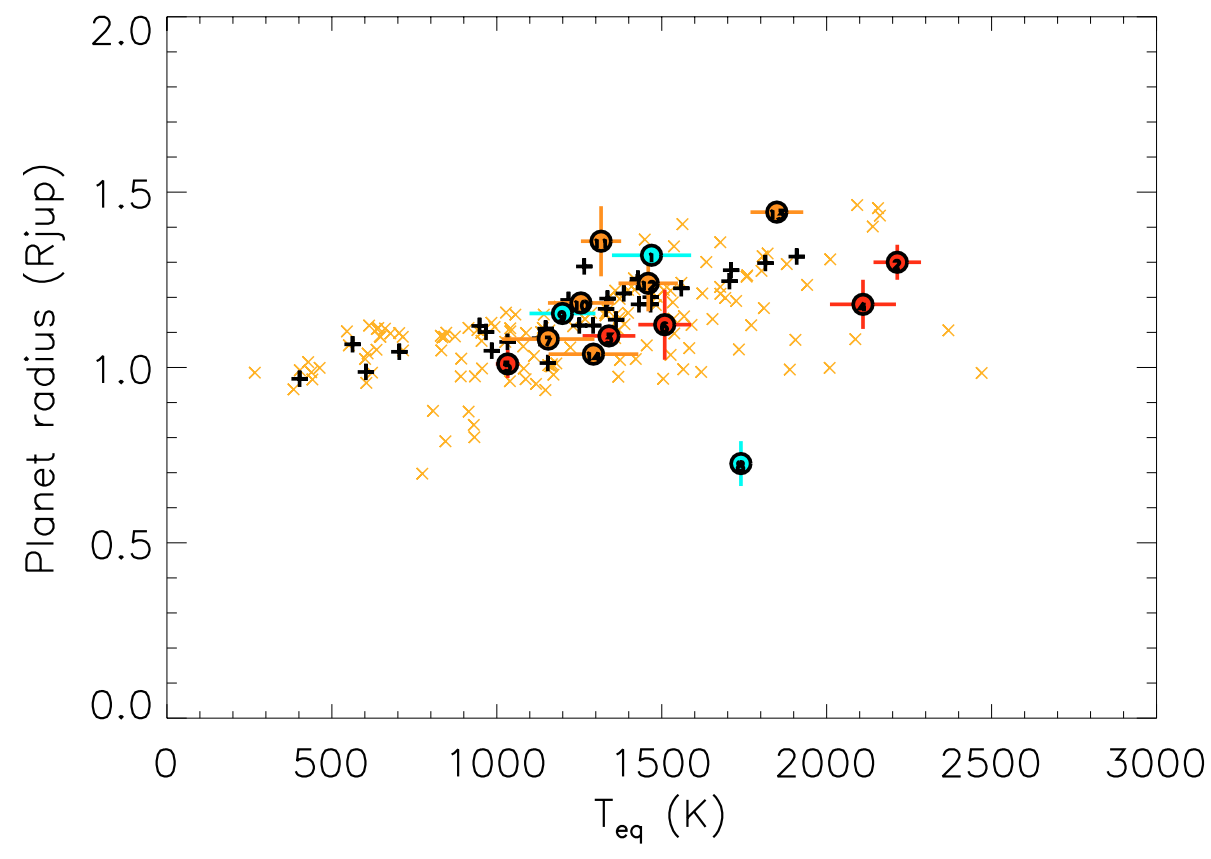

Fig. 15. Radius as a function of equilibrium temperature of transiting exoplanets. (OGLE planets are red circles, other transit surveys in orange, planets from radial velocity surveys in blue. Simulated planets detected: black plusses, under threshold: orange crosses). The OGLE planets equilibrium temperature-radius distribution is at $1.22 \sigma$ from the maximum likelihood of the model $(1.05 \sigma$ considering the 11 planets discovered by transit surveys and $2.25 \sigma$ considering the 14 known planets).
Results with our fiducial model are presented in Fig. 16. We find that planets with low masses (say, less than Jupiter's mass) can have either very large or very small radii, depending on whether they contain a significant mass in heavy elements or not. On the other hand, massive planets have radii which are comparatively better defined. This is mostly due to the fact that we assume a maximum mass of heavy elements of $100 M_{\oplus}$, a hypothesis that will be tested directly by the discovery of a few massive transiting planets.

Our model results once again agree well with the detections made by photometry. Importantly, the yellow crosses in Fig. 16 do not lie significantly below the black ones: we predict that future surveys will not discover a population of small-sized giant planets, at least for masses above that of Saturn.

The presence of planets with larger masses of heavy elements should remain marginal because otherwise they would have been detected by present-day surveys, Fig. 16 showing that planets below $1 R_{\text {Jup }}$ are already detectable, although in favorable cases (small radius of the primary and bright targets). Quantitatively, simulations in the OGLE fields indicate that if planets had radii uniformly distributed between 0.5 and $1.5 R_{\text {Jup }}$, $18.5 \%$ of the planets discovered by the survey would have radii below $1 R_{\text {Jup. }}$. This fraction is not negligible and is (although marginally) inconsistent with the sample of $0 / 11$ planets with $R<R_{\text {Jup }}$ discovered by transit surveys thus far.

Therefore, although we cannot statistically rule out the presence of a population of small planets, these would require the formation of extremely metal-rich planets. Our prediction is a consequence of evolution models and of our assumption that planets with masses of heavy elements beyond $100 M_{\oplus}$ should be rare.

Figure 17 shows the ensemble of planets obtained for an extremely large number of draws, with our fiducial model. Voids in the ensemble of crosses correspond to the absence of planets with these masses in the radial-velocimetry list. They should not be considered as significant. The contours in the figure indicate the ensemble of masses and radii expected for planets with different masses of heavy elements, from 0 to $100 M_{\oplus}$. Importantly, the location of these contours is linked to our assumption of an energy source in the planetary interior equal to $0.5 \%$ times the irradiation received by the planet. Independently of the details of this assumption, this shows that a statistically significant ensemble of known transiting planets would allow a determination of the presence or lack of heavy elements in these objects.

We have also tested another assumption regarding the planetary evolution model: all planets possess $20 M_{\oplus}$ mass in heavy elements, $70 \%$ of them have no extra heat source, whereas $30 \%$ have $3 \times 10^{26} \mathrm{erg} \mathrm{s}^{-1}$ dissipated at the center. With this assumption, one can qualitatively explain the observed transiting planets (i.e. the "normal" planets and the "anomalously large" ones, respectively) with the exception of HD $149026 \mathrm{~b}$, for which one could argue that the planet comes from a different population. In this case, Fig. 18 shows a distribution of radii that is relatively similar to the previous one (Fig. 16), with the exception that no planet has a radius smaller than $0.8 R_{\mathrm{Jup}}$. In this case, the 2 regions corresponding to the "standard" model, and to the "heat dissipation" case are clearly different, especially at the low-mass range of the diagram.

Present observations cannot distinguish between the two models, showing the need for additional detections of transiting giant planets. Particularly important are planets between the mass of Saturn and that of Jupiter, as this is a mass regime where expected compositional differences have the largest impact.

\section{Conclusions}

We have presented a simulation of photometric transiting surveys based on basic knowledge of the stellar and planetary populations in the galactic neighborhood and on a planetary evolution model tuned to the information obtained from transiting giant planets with masses above that of Saturn. This simulation was applied to the OGLE survey, and shown to yield a generally excellent agreement with the transiting planets detected by the survey.

We have thus shown that radial velocimetry and photometric surveys are compatible within statistical uncertainties, in agreement with Gould et al. (2006). We have derived a frequency of very close-in planets with orbital periods shorter than 2 days around solar-type stars, of $(1 / 1265)\left(1_{-0.33}^{+0.33}\right)$ at a $60 \%$ confidence level or $(1 / 1265)\left(1_{-0.5}^{+0.83}\right)$ at a $90 \%$ confidence level. 

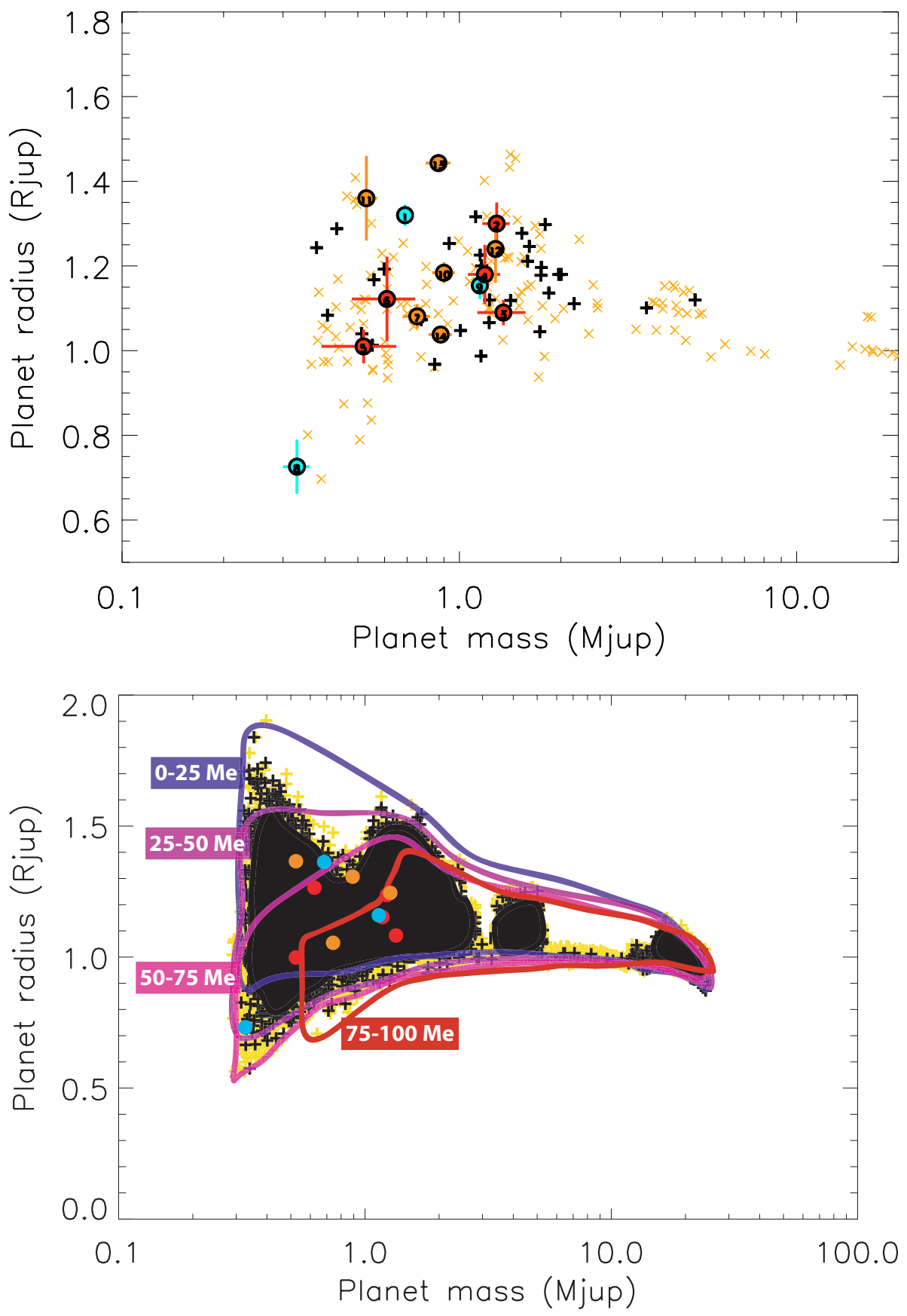

Fig. 16. Mass-radius relation for transiting extrasolar giant planets. (OGLE planets are red circles, other transit surveys in orange, planets from radial velocity surveys in blue. Simulated planets detected: black plusses, under threshold: orange crosses). The OGLE planets massradius distribution is at $0.67 \sigma$ from the maximum likelihood of the model $(0.72 \sigma$ considering the 11 planets discovered by transit surveys and $0.97 \sigma$ considering the 14 known planets).

Fig. 17. Mass-radius relation for a very large number of Monte-Carlo trials using the fiducial model. The curves show the ensemble of planets with masses of heavy elements between 0 and 25, 25 and 50, 50 and 75, 75 and $100 M_{\oplus}$, respectively. Symbols are as in Fig. 8.

Using null results by photometric surveys for given ranges of parameters, we are able to strengthen two results already present in the radial velocimetry data:

- Stars with low metallicities $([\mathrm{Fe} / \mathrm{H}]<-0.07)$ do not, or are very unlikely to harbour close-in giant planets with orbital periods $P<10$ days. This is unlike stars above that metallicity threshold (see Fig. 12).

- There is a lack of small-mass giant planets below the mass of Jupiter and above that of Saturn for orbital periods $P<$ 3 days (see Fig. 8).

Further data is required to precisely quantify these empirical results that bear important consequences for our understanding of planet formation and migration.

On the basis of our model, and assumptions concerning the composition of giant planets (i.e. masses of heavy elements between 0 and $100 M_{\oplus}$ ), we find that the present detections of transiting planets have sampled a population that is quite representative of the main population of giant planets, at least for the ones that are above about half the mass of Jupiter. We hence predict that future transit surveys with higher sensitivities will not discover a significant population of yet undetected Jupiter-mass planets with small sizes, i.e. radii smaller than that of Jupiter (see Fig. 16).

Many ground-based transit surveys are in progress, and with the space missions CoRoT (Baglin et al. 2002) and Kepler (Borucki et al. 2003), the number of known transiting planets is expected to rise rapidly over the next few years. This will enable us to better test the models and quantify some of the results presented in this article. We also hope to be able to discriminate between various models of the evolution and compositions of giant planets, a matter of great importance for formation models.

We wish to stress however that a continuation of groundbased transit surveys is desirable even in the presence of 


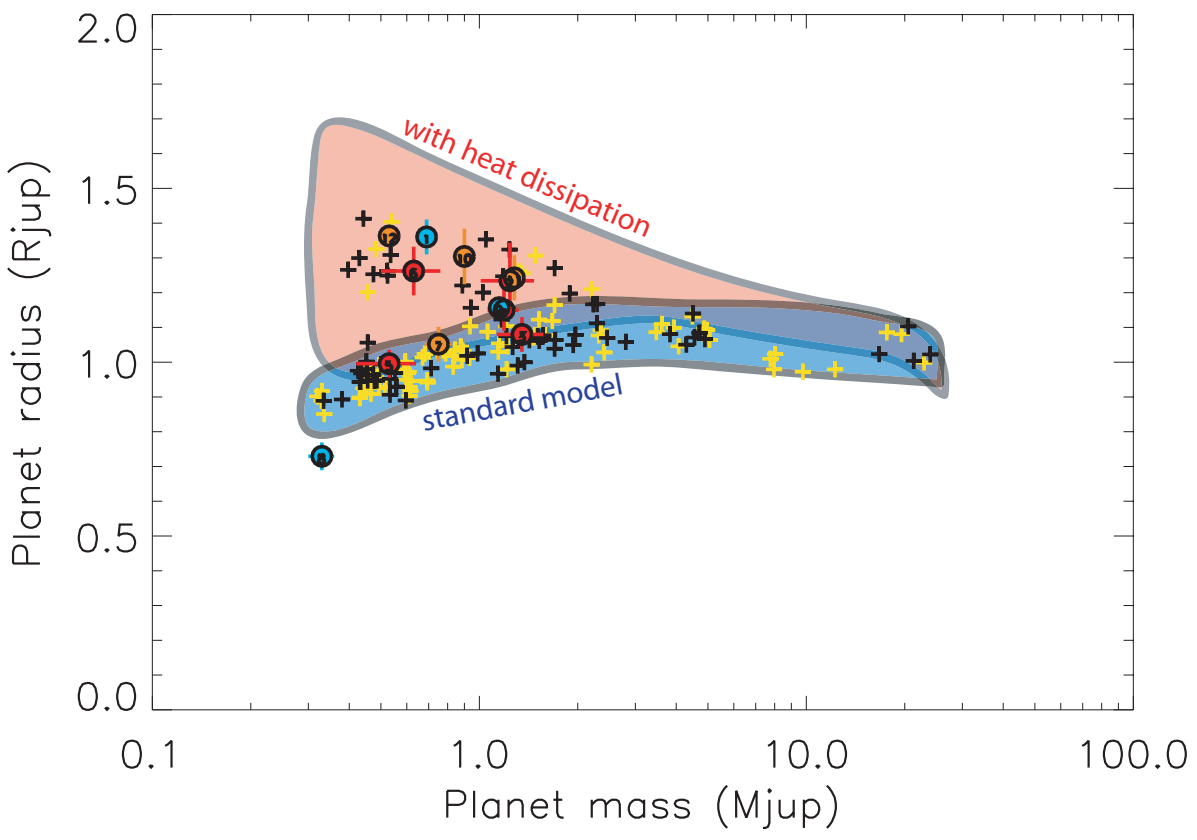

Fig. 18. Mass-radius relation obtained for an alternative model with $70 \%$ of "standard" planets with no extra-energy source, and $30 \%$ planets receiving an additional $3 \times 10^{26} \mathrm{erg} \mathrm{s}^{-1}$ luminosity dissipated at the center. All planets are assumed to possess $20 M_{\oplus}$ in heavy elements. Symbols are as in Fig. 8. similar programs from space. CoRoT will survey 60000 dwarf stars over five 150-day periods and Kepler about 100000 over 4 years, implying a maximum potential yield of 55 and 90 transiting giant planets, respectively, plus many other smaller planets. Concerning giant planets, quantifying the fraction of very close-in planets with a $10 \%$ accuracy at the $3 \sigma$ level would require the discovery of $\sim 200$ transiting planets. Understanding the evolution and compositions of giant planets will require an even larger number of detections. The radius of a giant planet itself depends mainly on four parameters: the planetary mass, equilibrium temperature, age, and its composition (note that the composition can be considered as a simple parameter only in the case of planets mostly made of hydrogen and helium: smaller planets will be more difficult to model!). Additional energy sources may occur (such as in the presence of tidal heat dissipation), and the initial conditions and formation history may have their say in the matter as well. Furthermore, the observational uncertainties are generally large. For example, the planetary radius is generally only known to $\sim 10 \%$, for a global variation that is relatively small ( 1 to $1.5 R_{\mathrm{Jup}}$ ). This implies that to constrain a given correlation to, say $10 \%$, and with four independant variables, hundreds of data points are needed, and thousands would be desirable.

This motivates us to seek programs capable of detecting thousands of transiting planets in the mid-term future, and ways to reduce the error bars on the different parameters. One direction is to test the Dome C plateau in Antarctica for such an ambitious program, which is the purpose of A STEP (Fressin et al. 2005). Other directions exist, such as proposals for similar surveys from space. In any case, it is most important that a statistically significant population of exoplanets be characterized for a better understanding of planet formation and our origins.

Acknowledgements. The code used for this work, CoRoTlux, was developped as part of the CoRoT science program by the authors with major contributions by Aurélien Garnier, Maxime Marmier, Martin Vannier, Suzanne Aigrain, and help from Claire Moutou, Stéphane Lagarde, Antoine Llebaria, Didier Queloz, and François Bouchy. We want to thank Andrzej Udalski and Michael Gillon for their communications on OGLE data, Frédéric Thévenin for his advice on stellar populations simulation. F.F. was funded by grants from the French Ministère de la Recherche and by the Société des Amis des Sciences. V.M. was funded by a grant from the C.N.R.S. This work used
Jean Schneider's exoplanet database www.exoplanet.eu, and the Besançon model of the Galaxy at physique.obs-besancon.fr/modele/ extensively. The planetary evolution models used for this work can be downloaded at www.obs-nice.fr/guillot/pegasids/.

\section{References}

Alard, C., \& Lupton, R. H. 1998, ApJ, 503, 325

Alonso, R., Brown, T. M., Torres, G., et al. 2004, ApJ, 613, L153

Andrievsky, S. M., Luck, R. E., Martin, P., \& Lépine, J. R. D. 2004, A\&A, 413, 159

Baglin, A., Auvergne, M., Barge, P., et al. 2002, in ESA SP-485: Stellar Structure and Habitable Planet Finding, ed. B. Battrick, F. Favata, I. W. Roxburgh, \& D. Galadi, 17

Bakos, G. A., Noyes, R. W., Kovacs, G., et al. 2006, ArXiv Astrophysics e-prints Baraffe, I., Chabrier, G., Barman, T. S., et al. 2005, A\&A, 436, L47 Bodenheimer, P., Lin, D. N. C., \& Mardling, R. A. 2001, ApJ, 548, 466

Borucki, W. J., Koch, D. G., Lissauer, J. J., et al. 2003, in Future EUV/UV and Visible Space Astrophysics Missions and Instrumentation, ed. J. C. Blades, O. H. W. Siegmund, Proc SPIE, 4854, 129

Bouchy, F., Pont, F., Santos, N. C., et al. 2004, A\&A, 421, L13

Bouchy, F., Pont, F., Melo, C., et al. 2005, A\&A, 431, 1105

Brown, T. M. 2003, ApJ, 593, L125

Brown, T. M., Charbonneau, D., Gilliland, R. L., Noyes, R. W., \& Burrows, A. 2001, ApJ, 552, 699

Butler, R. P., Wright, J. T., Marcy, G. W., et al. 2006, ApJ, 646, 505

Charbonneau, D., Winn, J. N., Latham, D. W., et al. 2006, ApJ, 636, 445

Cody, A. M., \& Sasselov, D. D. 2002, ApJ, 569, 451

Collier Cameron, A., Bouchy, F., Hebrard, G., et al. 2006, ArXiv Astrophysics e-prints

Deming, D., Seager, S., Richardson, L. J., \& Harrington, J. 2005, Nature, 434, 740

Duquennoy, A., \& Mayor, M. 1991, A\&A, 248, 485

Fischer, D. A., \& Valenti, J. 2005, ApJ, 622, 1102

Fressin, F., Guillot, T., Bouchy, F., et al. 2005, in EAS Pub. Ser., ed. M. Giard, F. Casoli, \& F. Paletou, 309

Gillon, M., Courbin, F., Magain, P., \& Borguet, B. 2005, A\&A, 442, 731

Gillon, M., Pont, F., Moutou, C., et al. 2006, A\&A, 459, 249

Gonzalez, G. 1998, A\&A, 334, 221

Gould, A., Dorsher, S., Gaudi, B. S., \& Udalski, A. 2006, Acta Astron., 56, 1

Guillot, T. 2005, Ann. Rev. Earth Planet. Sci., 33, 493

Guillot, T., \& Showman, A. P. 2002, A\&A, 385, 156

Guillot, T., Santos, N. C., Pont, F., et al. 2006, A\&A, 453, L21

Holman, M. J., Winn, J. N., Latham, D. W., et al. 2006, ApJ, 652, 1715

Horne, K. 2001, in Techniques for the detection of planets and life beyond the solar system, 4th Annual ROE Workshop, held at Royal Observatory Edinburgh, Scotland, Nov 7-8, 2001, ed. W. R. F. Dent (Edinburgh, Scotland: Royal Observatory, Dent), 5

Irwin, J., Irwin, M., Aigrain, S., et al. 2007, MNRAS, 375, 1449 
Knutson, H. A., Charbonneau, D., Noyes, R. W., Brown, T. M., \& Gilliland, R. L. 2007, ApJ, 655, 564

Konacki, M., Sasselov, D. D., Torres, G., Jha, S., \& Kulkarni, S. R. 2003, BAAS, 1416

Konacki, M., Torres, G., Sasselov, D. D., et al. 2004, ApJ, 609, L37

Konacki, M., Torres, G., Sasselov, D. D., \& Jha, S. 2005, ApJ, 624, 372

Kovács, G., Zucker, S., \& Mazeh, T. 2002, A\&A, 391, 369

Laughlin, G., Wolf, A., Vanmunster, T., et al. 2005, ApJ, 621, 1072

Lecavelier des Etangs, A., Vidal-Madjar, A., McConnell, J. C., \& Hébrard, G. 2004, A\&A, 418, L1

Levrard, B., Correia, A. C. M., Chabrier, G., et al. 2007, A\&A, 462, L5

Lyons, L. 1986, Statistics for Nuclear and Particle Physics (Cambridge Univ. Press), 85

Magain, P., Courbin, F., Gillon, M., et al. 2007, A\&A, 461, 373

Marcy, G., Butler, R. P., Fischer, D., et al. 2005, Progr. Theor. Phys. Suppl., 158, 24

Mazeh, T., Zucker, S., \& Pont, F. 2005, MNRAS, 356, 955

McCullough, P. R., Stys, J. E., Valenti, J. A., et al. 2006, ApJ, 648, 1228

Moutou, C., Pont, F., Bouchy, F., \& Mayor, M. 2004, A\&A, 424, L31

Nordström, B., Mayor, M., Andersen, J., et al. 2004, A\&A, 418, 989

O'Donovan, F. T., Charbonneau, D., Mandushev, G., et al. 2006, ApJ, 651, L61

Pepper, J., \& Gaudi, B. S. 2005, ApJ, 631, 581

Pont, F., Bouchy, F., Queloz, D., et al. 2004, A\&A, 426, L15

Pont, F., Melo, C. H. F., Bouchy, F., et al. 2005, A\&A, 433, L21

Pont, F., Moutou, C., Bouchy, F., et al. 2006a, A\&A, 447, 1035
Pont, F., Zucker, S., \& Queloz, D. 2006b, MNRAS, 373, 231

Robin, A. C., Reylé, C., Derrière, S., \& Picaud, S. 2003, A\&A, 409, 523

Santos, N. C., Israelian, G., \& Mayor, M. 2004, A\&A, 415, 1153

Santos, N. C., Pont, F., Melo, C., et al. 2006, A\&A, 450, 825

Sato, B., Fischer, D. A., Henry, G. W., et al. 2005, ApJ, 633, 465

Showman, A. P. \& Guillot, T. 2002, A\&A, 385, 166

Shporer, A., Tamuz, O., Zucker, S., \& Mazeh, T. 2007, MNRAS, 136

Smith, A. M. S., Collier Cameron, A., Christian, D. J., et al. 2006, MNRAS, 373, 1151

Stetson, P. B. 1987, PASP, 99, 191

Tamuz, O., Mazeh, T., \& Zucker, S. 2005, MNRAS, 356, 1466

Torres, G., Konacki, M., Sasselov, D. D., \& Jha, S. 2003, in BAAS, 1235

Udalski, A. 2002, IAU Circ., 7925, 2

Udalski, A. 2003, Acta Astron., 53, 291

Udalski, A., Paczynski, B., Zebrun, K., et al. 2002, Acta Astron., 52, 1

Udalski, A., Szymanski, M. K., Kubiak, M., et al. 2004, Acta Astron., 54, 313

Udry, S., Fischer, D., \& Queloz, D. 2007, in Protostars and Planets V, ed. B. Reipurth, D. Jewitt, \& K. Keil (Tucson: University of Arizona Press), 951, 685

Wilson, D. M., Enoch, B., Christian, D. J., et al. 2006, PASP, 118, 1245

Winn, J. N. \& Holman, M. J. 2005, ApJ, 628, L159

Winn, J. N., Noyes, R. W., Holman, M. J., et al. 2005, ApJ, 631, 1215

Winn, J. N., Holman, M. J., \& Fuentes, C. I. 2007, AJ, 133, 11

Wittenmyer, R. A., Welsh, W. F., Orosz, J. A., et al. 2005, ApJ, 632, 1157

Zucker, S. \& Mazeh, T. 2001, ApJ, 562, 1038 